\title{
Quantizing higher-spin gravity in free-field variables
}

\author{
Andrea Campoleoni, ${ }^{a}$ Stefan Fredenhagen ${ }^{b, c}$ and Joris Raeymaekers ${ }^{d}$ \\ ${ }^{a}$ Institut für Theoretische Physik, ETH Zurich, \\ Wolfgang-Pauli-Strasse 27, 8093 Zürich, Switzerland \\ ${ }^{b}$ University of Vienna, Faculty of Physics, \\ Boltzmanngasse 5, 1090 Vienna, Austria \\ ${ }^{c}$ Erwin Schrödinger International Institute for Mathematics and Physics, \\ University of Vienna, \\ Boltzmanngasse 9, 1090 Vienna, Austria \\ ${ }^{d}$ CEICO, Institute of Physics of the ASCR, \\ Na Slovance 2, 18221 Prague 8, Czech Republic \\ E-mail: campoleoni@itp.phys.ethz.ch, stefan.fredenhagen@univie.ac.at, \\ joris@fzu.cz
}

ABSTRACT: We study the formulation of massless higher-spin gravity on $\mathrm{AdS}_{3}$ in a gauge in which the fundamental variables satisfy free field Poisson brackets. This gauge choice leaves a small portion of the gauge freedom unfixed, which should be further quotiented out. We show that doing so leads to a bulk version of the Coulomb gas formalism for $W_{N}$ CFT's: the generators of the residual gauge symmetries are the classical limits of screening charges, while the gauge-invariant observables are classical $W_{N}$ charges.

Quantization in these variables can be carried out using standard techniques and makes manifest a remnant of the triality symmetry of $W_{\infty}[\lambda]$. This symmetry can be used to argue that the theory should be supplemented with additional matter content which is precisely that of the Prokushkin-Vasiliev theory. As a further application, we use our formulation to quantize a class of conical surplus solutions and confirm the conjecture that these are dual to specific degenerate $W_{N}$ primaries, to all orders in the large central charge expansion.

Keywords: AdS-CFT Correspondence, Conformal and W Symmetry, Field Theories in Lower Dimensions, Higher Spin Gravity

ARXIV EPRINT: 1712.08078 


\section{Contents}

1 Introduction 1

2 Review of higher-spin gravity in Chern-Simons formulation 3

2.1 Chern-Simons theory with boundary 3

$\begin{array}{lll}2.2 & \text { AdS asymptotic conditions and Drinfeld-Sokolov gauges } & 6\end{array}$

$\begin{array}{lll}3 & \text { The diagonal gauge } & 7\end{array}$

3.1 Reaching the diagonal gauge 8

$\begin{array}{llr}3.2 & \text { Residual gauge symmetries } & 9\end{array}$

3.3 Gauge orbits 12

4 Asymptotic symmetries and quantization $\quad \mathbf{1 5}$

4.1 Global symmetries and Poisson brackets of free fields 16

$\begin{array}{lll}4.2 & \text { Screening charges as generators of residual gauge symmetries } & 18\end{array}$

$\begin{array}{lll}4.3 & \text { Classical } W_{N} \text { algebra } & 20\end{array}$

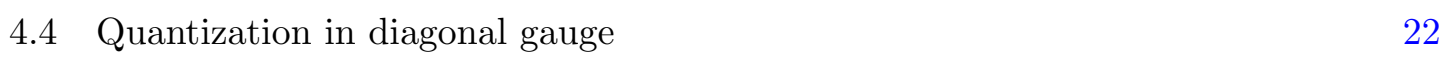

5 Application: quantization of conical solutions $\quad 24$

$\begin{array}{ll}5.1 & \text { Winding sectors and conical solutions } 24\end{array}$

$\begin{array}{lll}5.2 & \text { Classical symmetries and null vectors } & 26\end{array}$

$\begin{array}{ll}\text { 5.3 Quantization of conical solutions } & 27\end{array}$

$\begin{array}{llr}6 & \text { Discussion and outlook } & 29\end{array}$

$\begin{array}{ll}\text { A Gravity subsector } & 31\end{array}$

B Infinitesimal symmetries of Miura transform 32

C Null vectors and their classical limits 33

D All degenerate primaries as momentum-winding states $\quad 34$

\section{Introduction}

The AdS/CFT correspondence offers a nonperturbative definition of quantum gravity in anti-de Sitter space in the form of a dual conformal field theory (CFT), thus making conceptual problems in quantum gravity accessible, at least in principle. Being a strongweak coupling duality, a complete proof of the correspondence may be out of reach in the richest examples, though one may hope to do better in the recently uncovered simpler 
and more tractable instances of holographic duality. One promising class of dualities was identified by Gaberdiel and Gopakumar [1], who proposed that a (2+1)-dimensional higherspin gravity theory due to Prokushkin and Vasiliev [2] is holographically dual to a certain large $N$ limit of the $W_{N}$ minimal model CFT's. The bulk theory allows for a consistent truncation to the massless higher-spin sector which does not contain any locally propagating fields and can be elegantly described as a Chern-Simons theory. The only degrees of freedom in this sector are boundary fluctuations, which are furthermore controlled by a large $W_{\infty}[\lambda]$ symmetry.

Far from giving a complete proof of the higher-spin/minimal-model duality, we will in this work study a set of variables for the massless higher-spin sector in which quantization is relatively straightforward; these variables can roughly be thought of as Darboux coordinates on the phase space of higher-spin fields. For simplicity, we restrict our attention to the theory with higher-spin algebra $\operatorname{sl}(N, \mathbb{C})$ which describes spins $2, \ldots, N$ in Euclidean signature. This theory is based on an $\operatorname{sl}(N, \mathbb{C})$ Chern-Simons gauge field with boundary conditions which impose asymptotic AdS behaviour of the field. These boundary conditions are preserved by a group of 'Drinfeld-Sokolov (DS) gauge symmetries', and quotienting these out ('DS reduction' [3]) leads to a theory with classical $W_{N}$ symmetry [4, 5].

In CFT's with quantum $W_{N}$ symmetry, the Coulomb gas formalism [6] (see also the review [7]) gives a realization of the $W_{N}$ currents as operators built out of $N-1$ free fields which commute with a set of screening operators. On the bulk dual side, it has also been known since [8] that there exists a gauge for the DS gauge symmetry of the higher-spin theory, the 'diagonal gauge', in which the Poisson brackets of the fundamental variables reduce to those of free fields. However it was also pointed out in [8] that this gauge choice suffers from ambiguities in that there are many diagonal gauge representations of the same physical configuration. Also, it was not clear how the screening charges, which are vital for obtaining a $W_{N}$ symmetry, appear in this gauge, and if they are related to the aforementioned ambiguity.

In this work, we will address these puzzles in detail. We will show that the ambiguities observed in [8] arise from the fact that the diagonal gauge does not fix the DS gauge symmetry completely; rather it leaves a finite-parameter family of residual DS gauge transformations. These should be viewed as proper gauge transformations which do not change the physical state. ${ }^{1}$ We will see that the charges which generate these residual DS transformations through Poisson brackets are precisely the classical limits of the screening charges of the Coulomb gas formalism for $W_{N}$ CFT's. In addition, the observables which are invariant under the residual DS gauge symmetry are precisely the classical $W_{N}$ currents. Therefore all the ingredients of the Coulomb gas formalism appear naturally when considering bulk higher-spin theory in the diagonal gauge.

Once the theory is formulated in free field variables, quantization can be carried out using standard techniques, and we will see that it leads to a quantum shift in the background charge of the free fields. This uncovers a $\mathbb{Z}_{2}$ duality symmetry which is not visible at the

\footnotetext{
${ }^{1}$ This is the main difference with respect to the formally similar gauge choice of $[9,10]$. In the latter context, the boundary conditions have been designed such that all residual symmetries are actual global symmetries, thus leading e.g. to a theory with a different asymptotic symmetry algebra with respect to that obtained with a DS reduction.
} 
classical level and is a remnant of the triality symmetry of the $W_{\infty}[\lambda]$ algebra [11]. This symmetry can be used to argue, modulo some natural assumptions, that the theory should be supplemented with additional matter content which is precisely that of the ProkushkinVasiliev theory.

When investigating the effect of the residual DS gauge transformation, we find a qualitative difference depending on whether we consider 'generic' field configurations, in which the gauge field is allowed to have isolated singularities around which the holonomy is nontrivial, or the 'non-generic' case where the holonomy is trivial everywhere. In the former case, the residual DS gauge transformations are discrete, giving a realization of the symmetric group, while in the latter case they act as continuous transformations. To illustrate the power of the free field formulation, we address in detail the quantization of the latter class of configurations with trivial holonomy. These were considered before in [12] and were shown to describe a class of generalized conical surpluses, and in our variables they appear as winding modes of the free fields. These solutions were conjectured to be dual to a subclass of degenerate primaries of the quantum $W_{N}$ algebra in [12, 13], and evidence for this has accumulated $[14,15] .^{2}$ Upon quantizing these solutions in our framework, we are able to confirm this conjecture to all orders in the $1 / c$ expansion.

\section{Review of higher-spin gravity in Chern-Simons formulation}

In this section we recall the key features of the Chern-Simons formulation of Euclidean higher-spin gauge theories in three-dimensional anti-de-Sitter (AdS) background. In particular, we review the boundary conditions that select asymptotically-AdS field configurations, stressing that they can be formulated in many different, albeit equivalent, ways. We focus on boundary conditions belonging to the class of Drinfeld-Sokolov gauges and we confront them with the diagonal gauge that will be analysed in the following sections.

\subsection{Chern-Simons theory with boundary}

Higher-spin gauge theories in three-dimensional anti-de-Sitter background, in the absence of matter couplings, can be formulated as pure Chern-Simons gauge theories [17]. Here we focus on Euclidean theories involving spins $2,3, \ldots, N$, which are described by a gauge field $A$ taking values in the complex Lie algebra $\operatorname{sl}(N, \mathbb{C})$. For a large part of this work, where we consider the infinitesimal symmetries of the theory, the precise global structure of the gauge group will be immaterial. When global considerations will enter the discussion, notably in section 3.3 , we will assume the gauge group to be $\operatorname{SL}(N, \mathbb{C}) / \mathbb{Z}_{N}$. This means that, as in [12], we mod out by the center $\mathbb{Z}_{N}$, generated by the element $e^{2 \pi i / N} \mathbb{1}$ in the defining representation, which is invisible to adjoint fields.

The Euclidean action is

$$
I_{E}=-i\left(S_{C S}[A]-S_{C S}[\bar{A}]\right)=2 \operatorname{Im}\left(S_{C S}[A]\right),
$$

\footnotetext{
${ }^{2}$ The same states have been also recently considered in a related holographic scenario, where they have been identified in the twisted sector of symmetric orbifold CFT's, which have been conjectured to be dual to the tensionless limit of strings on $\mathrm{AdS}_{3}$ [16].
} 
where $S_{C S}[A]$ is the Chern-Simons action

$$
S_{C S}[A]=\frac{k}{4 \pi} \int_{\mathcal{M}} \operatorname{tr}_{\mathbf{N}}\left(A \wedge d A+\frac{2}{3} A \wedge A \wedge A\right) .
$$

In the formulae above, $k$ is a real parameter, $\operatorname{tr}_{\mathbf{N}}$ is the trace in the defining $N$-dimensional representation of $\operatorname{sl}(N, \mathbb{C})$, and $\bar{A}$ is the complex conjugate ${ }^{3}$ of $A$. See [18] for more details on the Chern-Simons formulation of Euclidean gravity and e.g. appendix A.1 of [19] for its higher-spin extensions. We only consider the case in which the spin-2 subsector corresponds to the principal embedding of $\operatorname{sl}(2, \mathbb{C})$ into $\operatorname{sl}(N, \mathbb{C})$. As shown in appendix A, with this choice the constant $k$ is related to the $\mathrm{AdS}_{3}$ radius $l$ and the $3 \mathrm{D}$ Newton constant $G_{N}$ as

$$
k=-\frac{3 l}{2 N\left(N^{2}-1\right) G_{N}} .
$$

Note that, in our conventions, the weakly coupled gravity regime corresponds to taking $k$ large and negative.

To simplify the comparison of our ensuing analysis with CFT techniques, we assume the manifold $\mathcal{M}$ to have the topology of a solid sphere. The boundary $S^{2}$ corresponds to the usual cylindrical boundary of $A d S_{3}$ where we added the two points at infinity. In actual computations, following the most common CFT setup, we will perform an additional conformal transformation and describe the boundary as a plane parameterised by a complex coordinate $z$. In these conventions, Euclidean $A d S_{3}$ can be parameterised e.g. as $d s^{2}=$ $d \rho^{2}+e^{2 \rho} d z d \bar{z}$, where $\rho$ is a radial coordinate transverse to the boundary, while slices of constant Euclidean time are circles of constant $|z|$.

Upon varying the action (2.1) one picks up a boundary term:

$$
\delta S_{C S}=-\frac{k}{4 \pi} \int_{\partial \mathcal{M}} \operatorname{tr}_{\mathbf{N}}(A \wedge \delta A)+(\mathrm{eom}) .
$$

To have a good variational principle, we make it vanish by imposing the boundary conditions

$$
\left.\left(A_{\bar{z}}\right)\right|_{\partial \mathcal{M}}=0,\left.\quad\left(\bar{A}_{z}\right)\right|_{\partial \mathcal{M}}=0 .
$$

For $N>2$ these constraints are too restrictive to accommodate black holes [20], but they still allow to identify the asymptotic symmetries of the full space of solutions of the equations of motion $[4,5,19]$. Moreover, they are satisfied by the solutions we will discuss in section 5 , so that we stick to this simplifying choice. Actually, we further constrain the gauge connections to be of the form

$$
A=b^{-1} a_{z}(z) b d z+b^{-1} d b, \quad \bar{A}=b^{-1} \bar{a}_{\bar{z}}(\bar{z}) b d \bar{z}+b^{-1} d b,
$$

where $b=b(\rho)$ is a group element depending only on the coordinate $\rho$ transverse to the boundary. As discussed e.g. in [5, 21], this form of the connection can be reached by combining a residual gauge fixing with the equations of motion.

\footnotetext{
${ }^{3}$ Another form of the Euclidean action, which often appears in the literature, is given by $I_{E}=-i\left(S_{C S}[A]-S_{C S}\left[-A^{\dagger}\right]\right)$, where $\dagger$ stands for Hermitean conjugation. This form is equivalent to ours, since one can easily show that $S_{C S}\left[-A^{\dagger}\right]=S_{C S}[\bar{A}]$.
} 
Concretely, we take $b=e^{\rho J_{0}}$, where $J_{0}$ is a generator of the $\operatorname{sl}(2)$ subalgebra which is principally embedded in $\operatorname{sl}(N)$. For later convenience, we give an explicit representation for the generators of this $\operatorname{sl}(2)$ :

$$
J_{0}=\sum_{i=1}^{N}\left(\frac{N+1}{2}-i\right) e_{i i}, \quad J_{1}=-\sum_{i=1}^{N-1} e_{i+1, i}, \quad J_{-1}=\sum_{i=1}^{N-1} i(N-i) e_{i, i+1},
$$

where the $e_{i j}$ are $N$-dimensional matrices with entries $\left(e_{i j}\right)_{k}{ }^{l}=\delta_{i k} \delta_{j}{ }^{l}$. These represent the algebra $\left[J_{m}, J_{n}\right]=(m-n) J_{m+n}$.

The gauge choice (2.6) is invariant under residual 'gauge' transformations with parameters $\Lambda=b^{-1} \lambda(z) b$ and $\bar{\Lambda}=b^{-1} \bar{\lambda}(\bar{z}) b$, which induce infinitesimal shifts of $a_{z}$ and $\bar{a}_{\bar{z}}$ of the form

$$
\delta a_{z}=\partial_{z} \lambda+\left[a_{z}, \lambda\right], \quad \delta \bar{a}_{\bar{z}}=\partial_{\bar{z}} \bar{\lambda}+\left[\bar{a}_{\bar{z}}, \bar{\lambda}\right],
$$

where $\lambda(z) \in \operatorname{sl}(N)$ is a purely holomorphic Lie algebra element. These transformations must actually be interpreted as global symmetries [22], whose associated conserved charges can be derived as follows (see e.g. [5, 21] for more details). The canonical generator of gauge transformations in the Chern-Simons theory is

$$
G(\Lambda)=\frac{k}{4 \pi} \int_{\Sigma} d x^{i} \wedge d x^{j} \operatorname{tr}_{\mathbf{N}}\left(\Lambda F_{i j}\right)+Q(\Lambda),
$$

where $F_{\mu \nu}=\partial_{\mu} A_{\nu}-\partial_{\nu} A_{\mu}+\left[A_{\mu}, A_{\nu}\right]$ is the field strength, while the $x^{i}$ are the coordinates on a slice of fixed time that we denoted by $\Sigma . Q(\Lambda)$ is a boundary term, whose role is to cancel the boundary contribution produced by the functional variation of $G(\Lambda)$ that determines its Poisson bracket with the fields:

$$
\delta G(\Lambda)=-\frac{k}{2 \pi} \int_{\Sigma} d x^{i} \wedge d x^{j} \operatorname{tr}_{\mathbf{N}}\left(\left(\partial_{i} \Lambda+\left[A_{i}, \Lambda\right]\right) \delta A_{j}\right)+\left(\frac{k}{2 \pi} \int_{\partial \Sigma} d x^{i} \operatorname{tr}_{\mathbf{N}}\left(\Lambda \delta A_{i}\right)+\delta Q(\Lambda)\right) .
$$

For field-independent gauge parameters one can readily integrate the variation $\delta Q(\Lambda)$ that guarantees the cancellation of the terms between brackets. In our conventions, one can rewrite the ensuing charges as contour integrals in the $z$-plane:

$$
Q(\lambda, \bar{\lambda})=-\frac{k}{2 \pi} \oint \operatorname{tr}_{\mathbf{N}}\left(\lambda a_{z} d z+\bar{\lambda} \bar{a}_{\bar{z}} d \bar{z}\right) .
$$

The integration contour is originally a counterclockwise circle of constant Euclidean time $|z|$, which of course can be deformed as long as no singularities of the integrand are encountered.

The charges (2.11) play the dual role of being conserved through the Noether theorem, but also of generating the associated global symmetries through the Dirac bracket as

$$
\delta a_{z}=\left\{Q(\lambda, \bar{\lambda}), a_{z}\right\}
$$

and its analogue for $\bar{a}_{\bar{z}}[22]$. This relation actually provides an efficient way to compute the Dirac bracket of the fields. Expanding them as

$$
a_{z}(z)=\sum_{m \in \mathbb{Z}} a_{m} z^{-m-1}, \quad \bar{a}_{\bar{z}}=\sum_{m \in \mathbb{Z}} \bar{a}_{m} \bar{z}^{-m-1}
$$


and introducing arbitrary elements $\alpha, \beta$ of $\operatorname{sl}(N)$, by substituting (2.8) and (2.11) in (2.12) one obtains the affine $\operatorname{sl}(N)$ Lie algebra

$$
\left\{\operatorname{tr}_{\mathbf{N}}\left(\alpha a_{m}\right), \operatorname{tr}_{\mathbf{N}}\left(\beta a_{n}\right)\right\}=\frac{i}{k}\left(\operatorname{tr}_{\mathbf{N}}\left([\alpha, \beta] a_{m+n}\right)+m \operatorname{tr}_{\mathbf{N}}(\alpha \beta) \delta_{m+n, 0}\right)
$$

together with its analogue for $\bar{a}_{\bar{z}}$.

\subsection{AdS asymptotic conditions and Drinfeld-Sokolov gauges}

Selecting AdS asymptotic behaviour requires additional constraints on the strictly lowertriangular part $\left(a_{z}\right)_{+}$of the gauge potential $a_{z}[4,5,21]$ :

$$
\left(a_{z}\right)_{+} \approx J_{1}
$$

where $J_{1}$ is one of the generators of the $\mathrm{sl}(2)$ subalgebra defined in $(2.7)$. The symbol $\approx$ stresses that we are imposing a constraint on the phase space of the boundary degrees of freedom of the Chern-Simons theory with boundary conditions (2.5). This is a first class constraint: indeed it can be formulated as

$$
\operatorname{tr}_{\mathbf{N}}\left(\alpha_{-}\left(a_{m}-J_{1} \delta_{m,-1}\right)\right) \approx 0
$$

for all strictly upper-triangular (constant) matrices $\alpha_{-}$, which gives

$$
\left\{\operatorname{tr}_{\mathbf{N}}\left(\alpha_{-}\left(a_{m}-J_{1} \delta_{m,-1}\right)\right), \operatorname{tr}_{\mathbf{N}}\left(\beta_{-}\left(a_{n}-J_{1} \delta_{n,-1}\right)\right)\right\}=\frac{i}{k} \operatorname{tr}_{\mathbf{N}}\left(\left[\alpha_{-}, \beta_{-}\right] a_{m+n}\right)
$$

thanks to (2.14). The right-hand side is again proportional to the constraint because the commutator of two strictly upper-triangular matrices has only entries starting from the second upper diagonal, and therefore

$$
\operatorname{tr}_{\mathbf{N}}\left(\left[\alpha_{-}, \beta_{-}\right] a_{m+n}\right)=\operatorname{tr}_{\mathbf{N}}\left(\left[\alpha_{-}, \beta_{-}\right]\left(a_{m+n}-J_{1} \delta_{m+n,-1}\right)\right) .
$$

This result allows one to interpret the transformations (2.8) preserving the constraint (2.15), which are generated by arbitrary strictly upper-triangular matrices

$$
\lambda(z)=\lambda_{-}(z),
$$

as a proper gauge symmetry.

The upper-triangular gauge redundancy (2.19) can be eliminated by imposing suitable gauge-fixing conditions. Most prevalent in the literature are those belonging to the class of Drinfeld-Sokolov gauges $[3,8]$. These fix the residual gauge symmetry completely, so that the full set of constraints becomes second class. Let us illustrate the situation in the case of $\operatorname{sl}(2)$. We expand the modes $a_{m}$ of $a_{z}$ in the generators $J_{i}$ of $\operatorname{sl}(2)$,

$$
a_{m}=\sum_{i=-1}^{1} a_{m}^{i} J_{i} .
$$

The constraint (2.15) then reads

$$
\mathcal{C}_{m}=a_{m}^{1}-\delta_{m,-1} \approx 0
$$


and the (in this case unique) Drinfeld-Sokolov gauge-fixing condition is

$$
\mathcal{G}_{m}=a_{m}^{0} \approx 0 .
$$

The constraints satisfy the Poisson brackets

$$
\begin{aligned}
\left\{\mathcal{C}_{m}, \mathcal{G}_{n}\right\} & =-\frac{2 i}{k} a_{m+n}^{1} \approx-\frac{2 i}{k} \delta_{m+n,-1}, \\
\left\{\mathcal{G}_{m}, \mathcal{G}_{n}\right\} & =\frac{2 i m}{k} \delta_{m+n, 0}, \\
\left\{\mathcal{C}_{m}, \mathcal{C}_{n}\right\} & =0,
\end{aligned}
$$

so that the constraint matrix is invertible. The constraints are therefore second class, which confirms that $\mathcal{G}_{m}$ is a complete gauge fixing.

In this work we consider another gauge which is outside of the Drinfeld-Sokolov class. In the $\operatorname{sl}(2)$ case it is specified by the partial gauge-fixing condition

$$
\mathcal{D}_{m}=a_{m}^{-1} \approx 0 .
$$

The constraint matrix has entries

$$
\begin{aligned}
\left\{\mathcal{C}_{m}, \mathcal{D}_{n}\right\} & =-\frac{i}{k}\left(a_{m+n}^{0}+m \delta_{m+n, 0}\right), \\
\left\{\mathcal{D}_{m}, \mathcal{D}_{n}\right\} & =0 \\
\left\{\mathcal{C}_{m}, \mathcal{C}_{n}\right\} & =0
\end{aligned}
$$

and it is not invertible on the whole constraint surface due to the dependence on $a^{0}$. An example of a configuration on which invertibility fails is when

$$
a_{m}^{0}=-\Lambda \delta_{m}^{0}, \quad \text { with } \Lambda \in \mathbb{Z} .
$$

One sees from (2.25) that on this configuration, $\mathcal{C}_{\Lambda}$ and $\mathcal{D}_{-\Lambda}$ remain as first class constraints. These configurations and their $\operatorname{sl}(N)$ generalizations will play an important role in what follows.

The generalisation of the partial gauge-fixing (2.24) to $\operatorname{sl}(N)$ amounts to constraining the strictly upper-triangular part of the fields $a_{z}$ to vanish, i.e.

$$
\left(a_{z}\right)_{-} \approx 0,
$$

which we will refer to as the 'diagonal gauge'. The subtleties associated to this gauge choice will be discussed in the next two sections.

\section{The diagonal gauge}

In this section we study the formulation of higher-spin gravity in the diagonal gauge (2.27). As we shall see, this gauge has the great advantage that the Poisson brackets of the basic fields become extremely simple: they are those of free fields. On the other hand, it is not a Drinfeld-Sokolov gauge and this leads to some puzzles which were first anticipated in [8] 
and which we aim to address in this work. They stem from the property we already noted above in the $N=2$ case, namely that the diagonal gauge does not completely fix the proper gauge freedom (2.19). After discussing the origin of these residual gauge symmetries, we study the corresponding gauge orbits in detail. This paves the way for the discussion, in the next section, of how to factor out the residual symmetries in order to obtain a theory equivalent to the one formulated in a Drinfeld-Sokolov gauge.

\subsection{Reaching the diagonal gauge}

In the diagonal gauge the nontrivial components of the connection reside on the main diagonal, so that it can be parameterised as follows:

$$
a_{z}=J_{1}+\frac{1}{\sqrt{k}} \sum_{j=1}^{N} \vec{\epsilon}_{j} \cdot\left(i \partial_{z} \vec{\phi}\right) e_{j j} .
$$

Here, $\partial_{z} \phi^{a}(z)$, with $a=1, \ldots, N-1$, form a vector of purely $z$-dependent fields (the arrow notation $\vec{v}$ will be used to denote $(N-1)$-component vectors). We will impose also some regularity conditions on the fields $\partial_{z} \phi^{a}(z)$, which we shall spell out when they become relevant for the discussion, namely in section 3.3. The set of $N$ vectors $\vec{\epsilon}_{i}$, with $i=1, \ldots, N$, are instead the weights of the vector representation of $\operatorname{sl}(N)$. They satisfy the properties (see e.g. section 6.3.3 of [23])

$$
\vec{\epsilon}_{i} \cdot \vec{\epsilon}_{j}=\delta_{i j}-\frac{1}{N}, \quad \sum_{i=1}^{N} \vec{\epsilon}_{i}=0, \quad \sum_{i} \epsilon_{i a} \epsilon_{i b}=\delta_{a b} .
$$

We recall for later use the relation between the $\vec{\epsilon}_{i}$ and simple roots $\vec{e}_{a}$ and fundamental weights $\vec{\omega}_{a}$ of $\operatorname{sl}(N)$ :

$$
\vec{\omega}_{a}=\sum_{i=1}^{a} \vec{\epsilon}_{i}, \quad \vec{\epsilon}_{i}=\vec{\omega}_{i}-\vec{\omega}_{i-1}, \quad \vec{e}_{a}=\vec{\epsilon}_{a}-\vec{\epsilon}_{a+1} .
$$

According to (3.2), the roots are normalized to have length squared equal to 2 .

By taking the complex conjugate of (3.1) one obtains an analogous parameterisation of $\bar{a}_{\bar{z}}$ in terms of the antiholomorphic fields $\partial_{\bar{z}} \bar{\phi}^{a}(\bar{z})$; we will usually display only the expressions for the holomorphic sector in what follows.

Locally, the gauge (3.1) is reachable by making a finite proper gauge transformation of the type (2.19) as for a Drinfeld-Sokolov gauge. To see this, we start from an arbitrary flat connection $a_{z}$ satisfying the constraint (2.15). We note that locally we can write the gauge potential in pure gauge form, $a_{z}=g^{-1} \partial_{z} g$, with $g(z)$ a $z$-dependent element of the gauge group. Note that $g$ is determined only up to a left multiplication by a constant group element

$$
g(z) \rightarrow \tilde{\Lambda}_{0} g(z) .
$$

Finite gauge transformations arise from right multiplication by a $z$-dependent group element

$$
g(z) \rightarrow g(z) \Lambda(z)
$$


We now make a Gauss decomposition

$$
g=N_{+} h N_{-},
$$

where $h$ is a diagonal group element and $N_{+}$and $N_{-}$are lower and upper triangular group elements, respectively, whose diagonal entries are all equal to one. To reach the diagonal gauge, we make a finite upper-triangular gauge transformation with parameter $\Lambda=N_{-}^{-1}$ so that

$$
g=N_{+} h
$$

In this gauge, the asymptotic AdS condition (2.15) is satisfied for arbitrary $h$ and for $N_{+}$ determined in terms of $h$, up to multiplication from the left by a constant matrix $\tilde{\Lambda}_{0}$, by the equations

$$
N_{+}^{-1} \partial_{z} N_{+}=h J_{1} h^{-1} .
$$

The form (3.1) then arises from choosing the following parameterisation of $h$ :

$$
h(z)=\sum_{j=1}^{N} \exp \left(\frac{1}{\sqrt{k}} \vec{\epsilon}_{j} \cdot(i \vec{\phi}(z))\right) e_{j j} .
$$

We should stress that the above discussion was purely local, and that the group element $g(z)$ in (3.7) is not guaranteed to be globally defined. We will discuss the conditions under which $g(z)$ is well-defined and their significance in section 3.3.

\subsection{Residual gauge symmetries}

Though the above argument shows that we can always locally reach the diagonal gauge (3.1), the resulting representation turns out not to be unique, as was observed in [8]. The origin of this non-uniqueness lies in the fact that the diagonal gauge does not completely fix the proper upper triangular gauge freedom (2.19) but allows for a small (generated by a finite number of constant parameters) amount of residual gauge symmetry. Let us establish the existence of these symmetries first from the point of view of the gauge potential.

Given $a_{z}=J_{1}+\mathcal{D}$ in diagonal gauge ( $\mathcal{D}$ diagonal), we look for the proper gauge transformations, i.e. for matrices $\Lambda_{-}(z)=\mathbb{1}+\lambda_{-}(z)$ with $\lambda_{-}$a strictly upper triangular matrix, such that

$$
\tilde{a}_{z}=\Lambda_{-}^{-1} \partial \Lambda_{-}+\Lambda_{-}^{-1} a_{z} \Lambda_{-}=J_{1}+\tilde{\mathcal{D}},
$$

where here and hereafter we denote $\partial_{z}$ simply by $\partial$, and $\tilde{\mathcal{D}}$ is diagonal. This is equivalent to

$$
\tilde{\mathcal{D}}=\Lambda_{-}^{-1} \partial \Lambda_{-}+\left(\Lambda_{-}^{-1} J_{1} \Lambda_{-}-J_{1}\right)+\Lambda_{-}^{-1} \mathcal{D} \Lambda_{-} .
$$

All three terms on the right hand side are upper triangular, $\Lambda_{-}^{-1} \partial \Lambda_{-}$is even strictly upper triangular. The condition that the right hand side is diagonal is therefore equivalent to

$$
\Lambda_{-}^{-1} \partial \Lambda_{-}+\Lambda_{-}^{-1}\left(\left[J_{1}, \Lambda_{-}\right]+\mathcal{D} \Lambda_{-}\right)-\left.\left(\Lambda_{-}^{-1}\left(\left[J_{1}, \Lambda_{-}\right]+\mathcal{D} \Lambda_{-}\right)\right)\right|_{\text {diag }}=0
$$


where $\left.\right|_{\text {diag }}$ denotes the projection to the diagonal part. Multiplying this equation by $\Lambda_{-}$ from the left one arrives ${ }^{4}$ at the result

$$
\partial \Lambda_{-}+\left[a_{z}, \Lambda_{-}\right]-\Lambda_{-}\left(\left.\left[J_{1}, \Lambda_{-}\right]\right|_{\text {diag }}\right)=0 .
$$

This is a system of nonlinear (due to the last term) differential equations. For simplicity let us discuss the case $N=2$ in some detail, where

$$
a_{z}=\left(\begin{array}{cc}
\frac{1}{\sqrt{k}} i \epsilon_{1} \partial \phi & 0 \\
-1 & \frac{1}{\sqrt{k}} i \epsilon_{2} \partial \phi
\end{array}\right) \quad, \quad \Lambda_{-}=\left(\begin{array}{cc}
1 & \lambda_{1} \\
0 & 1
\end{array}\right)
$$

The condition (3.13) leads to the equation

$$
\partial \lambda_{1}+\frac{i}{\sqrt{k}} e_{1} \partial \phi \lambda_{1}+\lambda_{1}^{2}=0
$$

where, in agreement with (3.3), $e_{1}=\epsilon_{1}-\epsilon_{2}$. This is a special form of the Riccati equation (or an instance of Bernoulli's equation) which can be solved by introducing $1 / \lambda_{1}$ as a new variable, which turns it into a linear equation. The most general non-trivial solution is

$$
\lambda_{1}=\partial \log n_{1},
$$

where $n_{1}$ is any integral of $-e^{-\frac{i}{\sqrt{k}} e_{1} \phi}$, that is

$$
\partial n_{1}=-e^{-\frac{i}{\sqrt{k}} e_{1} \phi}
$$

The corresponding finite proper gauge transformation is given by

$$
i \partial \phi \rightarrow i \partial \phi+\sqrt{k} \partial \log n_{1} e_{1} .
$$

For a given $n_{1}$ we can get all other integrals as $n_{1}+c$ with a complex constant $c$. For large $c$ we obtain the infinitesimal transformation

$$
\delta(i \partial \phi)=-\frac{1}{c} \sqrt{k} e^{-\frac{i}{\sqrt{k}} e_{1} \phi} e_{1}+\cdots .
$$

In order to generalize this discussion to arbitrary $N$, it is useful to describe the residual gauge symmetries we just found also using the parameterisation of $a_{z}$ in terms of a group element $g(z)$ of the form (3.7). From this point of view, their existence may appear somewhat surprising, since the Gauss decomposition in (3.6) is unique for a generic group element $g$ (see e.g. [24], Ch. 3). We should keep in mind however that $g(z)$ is determined only up to a left multiplication by a constant group element $\tilde{\Lambda}_{0}$. To see if there are residual gauge symmetries, we should therefore verify whether there exist constant group elements $\tilde{\Lambda}_{0}$ and upper triangular group elements $\Lambda_{-}(z)$ with diagonal entries equal to 1 , such that

$$
\tilde{g}=\tilde{\Lambda}_{0} g \Lambda_{-}
$$

\footnotetext{
${ }^{4}$ Using that $\left.\left(\Lambda_{-}^{-1} A\right)\right|_{\text {diag }}=A_{\text {diag }}=\left.\left(A \Lambda_{-}\right)\right|_{\text {diag }}$ for upper triangular $A$.
} 
is lower triangular, i.e.

$$
(\tilde{g})_{-}=0
$$

Let us once again treat the case $N=2$ in detail. The group elements $h$ and $N_{+}$are in this case given by

$$
h=\left(\begin{array}{cc}
e^{i \frac{\epsilon_{1}}{\sqrt{k}} \phi} & 0 \\
0 & e^{i \frac{\epsilon_{2}}{\sqrt{k}} \phi}
\end{array}\right), \quad N_{+}=\left(\begin{array}{cc}
1 & 0 \\
n_{1} & 1
\end{array}\right)
$$

where $n_{1}$ is determined by (3.8) to be a solution of (3.17). We observe that the equation that determines the component $n_{1}$ of $N_{+}$coincides with the equation (3.17) that determines the finite gauge transformation. Working out equation (3.21) one finds that it is solved by ${ }^{5}$

$$
\tilde{\Lambda}_{0}=\left(\begin{array}{cc}
0 & 1 \\
-1 & 0
\end{array}\right) \equiv P_{12}, \quad \Lambda_{-}=\left(\begin{array}{cc}
1 & \partial\left(\log n_{1}\right) \\
0 & 1
\end{array}\right) .
$$

We note that $\tilde{\Lambda}_{0}$ is essentially a permutation matrix exchanging the first and second rows. From inspecting the diagonal elements of $\tilde{g}$ we find once again that the field $\partial \phi$ transforms under the residual gauge symmetry as (3.18).

This analysis for $N=2$ can now be straightforwardly generalised to find the residual gauge transformations for arbitrary $N$, where one finds that they are determined by the solutions of the differential equations (3.8). For example, taking $\tilde{\Lambda}_{0}$ to be the permutation matrix $P_{a, a+1}$ exchanging the $a$-th and $(a+1)$-th rows (with an appropriate sign as in (3.23)) and $\Lambda_{-}$the compensating upper triangular gauge parameter, we find the family of residual gauge transformations

$$
P_{a, a+1}: \quad i \partial \vec{\phi} \rightarrow i \partial \vec{\phi}+\sqrt{k} \partial\left(\log n_{a}\right) \vec{e}_{a}
$$

where the $n_{a}$ solve the equation

$$
\partial n_{a}=-e^{-\frac{i}{\sqrt{k}} \vec{e}_{a} \cdot \vec{\phi}}
$$

The corresponding infinitesimal transformations are

$$
\delta_{a}(i \partial \vec{\phi})=-\frac{1}{c_{a}} \sqrt{k} e^{-\frac{i}{\sqrt{k}} \vec{e}_{a} \cdot \vec{\phi}_{e_{a}}}+\cdots
$$

More generally, one finds solutions to (3.21) for $\tilde{\Lambda}_{0}$ any permutation matrix $P_{a b}, a<$ $b$. These can however be constructed by composing elementary transformations of the type (3.24), since for example $P_{a, a+2}=\left(P_{a+1, a+2}\right)^{-1} P_{a, a+1} P_{a+1, a+2}$.

The above analysis tells us that, naively, the diagonal gauge allows an $N(N-1) / 2-$ parameter family of residual proper transformations, depending on the integration constants contained in the solution of the system (3.8). However we have not verified whether, in expressions such as (3.24), the transformed fields are well-defined, i.e. sufficiently regular. This is what we are now going to investigate.

\footnotetext{
${ }^{5}$ This solution is not quite unique in that we are still free to multiply $\tilde{\Lambda}_{0}$ on the left by a constant lower triangular matrix, which does not influence the transformation of the field $\partial \phi$. Also, the function $n_{1}$ in $\Lambda_{-}$ can be any integral of (3.17), independently of the one chosen for $N_{+}$.
} 


\subsection{Gauge orbits}

In this section we want to get a clearer picture of the gauge orbits swept out by the residual gauge transformations in the diagonal gauge. In order to do so, we must first specify which are the field configurations we allow, and then investigate which residual transformations map allowed field configurations into each other. In what follows we will impose that the $\partial \phi^{a}$ are analytic on the Riemann sphere with only isolated singularities and no branch cuts. The physical motivation behind this assumption is that we want to exclude multi-valued fields, but allow for point-like sources. For generic field configurations in this class and generic choices of the parameters entering in the residual gauge transformations such as (3.24), the transformed fields contain branch cuts and are outside of the allowed field space. Therefore our task is to determine for which field configurations and gauge parameters the residual gauge transformations do act within our field space.

For simplicity of the discussion we will focus on the action of the residual gauge transformations on fields which have singularities only in the origin and infinity, i.e. the infinite past and future in radial time. The results of this analysis can be extended straightforwardly to more general configurations of singularities. We will distinguish between two classes of field configurations, for which the analysis of the allowed residual transformations is qualitatively different.

Generic fields: discrete orbits. Let us consider a generic field configuration $\partial \vec{\phi}$ with singularities only in the origin and at infinity. The exponentials $e^{-\frac{i}{\sqrt{k}} \vec{e}_{a} \cdot \vec{\phi}}$ typically have a branch cut running from $z=0$ to infinity, and we see from (3.26) that the corresponding infinitesimal transformation is obstructed. Nevertheless it is still possible that the finite transformation (3.24) is well-defined for a specific choice of $n_{a}$. We therefore ask whether there is any $n_{a}$ satisfying (3.17) such that $\partial \log n_{a}$ is again analytic on $\mathbb{C}$ without branch cuts and only with isolated singularities. It turns out that there is precisely one such solution, given by

$$
n_{a}=d_{a} \int_{z}^{e^{2 \pi i} z} e^{-\frac{i}{\sqrt{k}} \vec{e}_{a} \cdot \vec{\phi}(\tilde{z})} d \tilde{z}
$$

Here, the constant $d_{a}$ is chosen such that $n_{a}$ solves (3.17), but it drops out of the transformation (3.18). This means that for each fixed $a$, the residual gauge symmetry (3.24) is just a discrete $\mathbb{Z}_{2}$ symmetry. When these are combined they generate the symmetric group and relate $N$ ! solutions.

It is instructive, and will be useful for what follows, to work out how the symmetric group acts on the simple subclass of 'zero mode' solutions where the 1-form $\partial \vec{\phi}$ has simple poles in the origin and at infinity:

$$
i \partial \vec{\phi}=\frac{\vec{\alpha}_{0}}{z} .
$$

One finds from evaluating (3.27) that the $a$-th elementary residual symmetry (3.24) acts on the zero modes as

$$
\vec{\alpha}_{0} \rightarrow s_{a}\left(\vec{\alpha}_{0}-\sqrt{k} \vec{\rho}\right)+\sqrt{k} \vec{\rho}
$$


where $\vec{\rho}=\sum_{a} \vec{\omega}_{a}$ is the Weyl vector and $s_{a}$ is the $a$-th elementary Weyl reflection defined as

$$
s_{a}(\vec{\beta})=\vec{\beta}-\left(\vec{e}_{a} \cdot \vec{\beta}\right) \vec{e}_{a} .
$$

Therefore, the symmetric group acts as the Weyl group on the shifted zero mode $\vec{\alpha}_{0}^{\text {cyl }} \equiv$ $\vec{\alpha}_{0}-\sqrt{k} \vec{\rho}$. Note that this quantity can be interpreted as the zero mode after making the conformal transformation to the cylinder (see section 5), the shift arising from the background charge.

Smooth gauge fields: continuous orbits. In the subspace of field configurations where the exponentials $e^{-\frac{i}{\sqrt{k}} \vec{e}_{a} \cdot \vec{\phi}}$ have no branch cuts, there is no obstruction for the infinitesimal transformation (3.19), and the residual gauge transformations have the chance of being realised as a continuous symmetry. Note that this subspace is characterized by a quantization condition on the residue in the origin:

$$
\operatorname{Res}_{z \rightarrow 0}(i \partial \vec{\phi}) \in \sqrt{k} W
$$

where $W$ is the weight lattice.

The condition (3.31) can be rephrased in a more insightful way as requiring that the diagonal group element $h(z)$ in the Gauss decomposition (3.7) is single-valued in the gauge group $\operatorname{SL}(N, \mathbb{C}) / \mathbb{Z}_{N}$. Indeed, from (3.9) we find that single-valuedness of $h(z)$ as $z$ encircles the origin imposes the following condition on $\vec{\phi}$ :

$$
\vec{\epsilon}_{i} \cdot \vec{\phi}\left(e^{2 \pi i} z\right)=\vec{\epsilon}_{i} \cdot \vec{\phi}(z)+2 \pi \sqrt{k}\left(m_{i}-\frac{m}{N}\right),
$$

where $m_{i} \in \mathbb{Z}$ and $\sum_{i} m_{i}=m$. We allowed for $h(z)$ to come back to itself up to a phase $e^{2 \pi i m / N}, m \in \mathbb{Z}$, since we mod out by the $\mathbb{Z}_{N}$ center. Multiplying by $\vec{\epsilon}_{i}$ and summing over $i$ gives, using (3.2),

$$
\vec{\phi}\left(e^{2 \pi i} z\right)=\vec{\phi}(z)+2 \pi \sqrt{k} \sum_{i} m_{i} \vec{\epsilon}_{i}
$$

Using the second relation in (3.3), we see that the lattice spanned by vectors of the form $\sum_{i} m_{i} \vec{\epsilon}_{i}$ is nothing but the weight lattice. Therefore single-valuedness of $h(z)$ in the origin is equivalent to (3.31).

Though we saw that the condition (3.31) ensures that the infinitesimal residual transformations (3.19) are well-defined, it does not guarantee that this remains true for finite residual transformations such as (3.24), since branch cuts may appear at higher order. From inspecting (3.24) one easily sees that this leads to further conditions on the fields. Indeed, if $e^{-\frac{i}{\sqrt{k}} \vec{e}_{a} \cdot \vec{\phi}}$ has a non-trivial residue in 0 , then every solution $n_{a}$, as well as $\partial \log n_{a}$, will have a cut and the elementary residual transformation (3.24) is not well-defined. Requiring that also the more general residual transformations, which can be obtained by composing the elementary ones, are well-defined leads to the additional condition that there are no residues in the exponentials of the transformed fields, such as

$$
\left(n_{b}(\vec{\phi})\right)^{-C_{a b}} e^{-\frac{i}{\sqrt{k}} \vec{e}_{a} \cdot \vec{\phi}}
$$


where $C_{a b}$ is the Cartan matrix, and so on. Though we will not attempt to give a complete characterization of the resulting restrictions on field space in this work, it is not hard to see that the full set of extra conditions can be summarized as the requirement that the group element $N_{+}$in the Gauss decomposition (3.7) is single-valued. This follows from our observation in the previous subsection that the differential equations which determine the residual gauge parameters are the same as those determining the components of $N_{+}$. Indeed from the discussion around (3.20) it can be readily seen that for a single-valued $N_{+}$ and a given $\tilde{\Lambda}_{0}$, also $\Lambda_{-}$is single-valued.

In summary, we saw that the residual gauge transformations act as continuous symmetries on field configurations for which both $h$ and $N_{+}$, and hence the group element $g$ in (3.7), is single-valued. This condition can be interpreted more physically as a smoothness condition for the gauge field $a_{z}$, namely that the holonomy of $a_{z}$ around the origin is trivial in the gauge group $\mathrm{SL}(N, \mathbb{C}) / \mathbb{Z}_{N}$. Indeed, $a_{z}$ has trivial holonomy around the origin if

$$
\mathcal{P} \exp \oint_{S_{z}} A=g^{-1}\left(e^{2 \pi i} z\right) g(z)=e^{2 \pi i m / N} \cdot \mathbb{1}
$$

for some integer $m$, where $S_{z}$ is a circle centered at the origin and going through $z$. Trivial holonomy is therefore equivalent to having $g(z)$ single valued in $\operatorname{SL}(N, \mathbb{C}) / \mathbb{Z}_{N}$.

To summarize, we found that the orbits under residual gauge symmetries are rather different depending on whether

- the field configuration $\partial \vec{\phi}$ leads to a singular gauge potential $a_{z}$, as is generically the case. The residual gauge transformations then act discretely, instructing us to identify discrete points in field space. At such generic points the diagonal gauge suffers from a Gribov-type ambiguity [25]: the gauge-fixing slice intersects the gauge orbit of the generic field configuration $N$ ! times.

- the field configuration $\partial \vec{\phi}$ leads to a smooth gauge potential $a_{z}$, in the sense that the holonomy is trivial. The residual gauge symmetries act as continuous symmetries, instructing us to identify points on continuous orbits in field space. In this case a finite-parameter family of residual gauge symmetries generates a motion tangent to the diagonal gauge slice specified by (3.1).

In both situations we should quotient our field space by the action of the residual symmetries, and this will be the subject of the next section. We conclude this section with some further comments:

- It is instructive to note that the continuous residual gauge transformations typically introduce additional pole terms in the fields $\partial \vec{\phi}$. For example, starting from the configuration with a pole in the origin and satisfying (3.31), $i \partial \vec{\phi}=-\sqrt{k} \vec{\Lambda} / z$ with $\vec{\Lambda} \in W$, we find that the residual transformations (3.24) act as

$$
P_{a, a+1}: \quad i \partial \vec{\phi} \rightarrow i \partial \overrightarrow{\tilde{\phi}}=-\frac{\sqrt{k} \vec{\Lambda}}{z}+\frac{\sqrt{k}\left(\Lambda_{a}+1\right) z^{\Lambda_{a}} \vec{e}_{a}}{z^{\Lambda_{a}+1}-z_{0}^{\Lambda_{a}+1}}
$$


with $z_{0}$ an integration constant. The transformed field has additional poles; for example there is now also a pole in $z_{0}$ with residue $\sqrt{k} \vec{e}_{a}$, which satisfies (3.31).

Only when we let $z_{0} \rightarrow 0$, all the poles merge to a first order pole in the origin, with a residue which is transformed according to (3.29). On the weight vector $\vec{\Lambda}$, the transformation acts as a shifted Weyl reflection

$$
P_{a, a+1}: \quad \vec{\Lambda} \rightarrow s_{a} \cdot \vec{\Lambda} \equiv s_{a}(\vec{\Lambda}+\vec{\rho})-\vec{\rho} .
$$

with $s_{a}$ the $a$-th elementary Weyl reflection, see (3.30).

- One way ${ }^{6}$ to think about the quantization condition (3.31) is to view the scalar fields as being periodic with identifications

$$
\vec{\phi} \sim \vec{\phi}+2 \pi \sqrt{k} \vec{\Lambda}
$$

with any vector $\vec{\Lambda} \in W$ in the weight lattice. The value of the residue in (3.31) then labels the different winding sectors.

- Our analysis is reminiscent of the classification of the coadjoint orbits of the Virasoro group [26], where one distinguishes between generic orbits and exceptional ones by the presence of continuous symmetries. We will indeed see that the trivial holonomy condition on $a_{z}$ defines a generalization of the exceptional orbits to the case of $W_{N}$ symmetry. ${ }^{7}$ In the quantum theory, these will turn out to correspond to degenerate representations, while the generic case leads to nondegenerate ones.

\section{Asymptotic symmetries and quantization}

In the previous section we have seen that it is possible to describe asymptotically AdS configurations in the diagonal gauge, but that the latter entails residual gauge symmetries. To obtain a theory equivalent to one formulated in a Drinfeld-Sokolov gauge, one must therefore quotient out these residual symmetries. This naturally leads to a classical version of the Coulomb gas formalism for CFT's with $W_{N}$ symmetry developed in [7]: the generators of the residual gauge symmetries are precisely the screening charges of the Coulomb gas formalism. In order to illustrate this picture, in this section we proceed as follows: we first build a Poisson bracket on the space of boundary excitations, as described by the fields that appear on the diagonal of the gauge connection (3.1). In section 4.1 we show that, under reasonable assumptions, one obtains the Poisson bracket of free fields. In section 4.2 we then build the canonical generator of the residual gauge symmetries on this phase space, obtaining a classical analogue of the screening charges of the Coulomb gas formalism. To support our findings, in section 4.3 we also show that the transformations generated by the screening charges are symmetries of the Miura transform, which relates the diagonal gauge

\footnotetext{
${ }^{6}$ Note that this way of obtaining the quantization condition (3.31) from winding mode quantization for a periodic scalar is by no means unique. For example, in appendix D we discuss an alternate realization, where the periods lie in the rescaled root lattice in the presence of a constant $B$-field.

${ }^{7}$ See [27] and references therein for a discussion of $W_{N}$ coadjoint orbits.
} 
to a specific Drinfeld-Sokolov gauge. This means that the gauge invariant observables on the free field phase space are precisely the $W_{N}$ charges that one obtains in Drinfeld-Sokolov gauges, thus showing the equivalence of the two approaches. In section 4.4 we eventually show how the subtle classical analysis of the diagonal gauge pays off when moving to quantisation: the resulting boundary phase space can indeed be quantized with standard and efficient techniques.

\subsection{Global symmetries and Poisson brackets of free fields}

To identify the Poisson brackets of the basic fields we now examine the variations (2.8) of the connection which preserve the diagonal gauge (3.1). We aim to distinguish between global symmetries - which determine the structure of the boundary phase space as we have reviewed in section 2 - and proper gauge symmetries.

We recall that we can write $a_{z}$ as

$$
a_{z}=J_{1}+h^{-1} \partial_{z} h
$$

with $h$ defined in (3.9). If one rewrites the gauge parameter as

$$
\lambda=h^{-1} \tilde{\lambda} h
$$

the transformation of $a_{z}$ then reads

$$
\delta_{\lambda} a_{z}=\partial_{z} \lambda+\left[a_{z}, \lambda\right]=h^{-1}\left(\partial_{z} \tilde{\lambda}+\left[h J_{1} h^{-1}, \tilde{\lambda}\right]\right) h .
$$

The diagonal gauge condition (3.1) is therefore preserved if and only if the factor in brackets vanishes away from the main diagonal. In terms of the components $\tilde{\lambda}_{j, k}$ of the matrix $\tilde{\lambda}$, this leads to the conditions

$$
\partial_{z} \tilde{\lambda}_{j, k}=e^{-\frac{i}{\sqrt{k}} \vec{e}_{j-1} \cdot \vec{\phi}} \tilde{\lambda}_{j-1, k}-e^{-\frac{i}{\sqrt{k}} \vec{e}_{k} \cdot \vec{\phi}} \tilde{\lambda}_{j, k+1} \quad \text { for } j \neq k .
$$

These equations determine the $\tilde{\lambda}_{j, k}$ for $j \neq k$ in terms of the elements on higher diagonals, while the elements on the main diagonal are arbitrary. Eqs. (4.4) therefore do not mix the lower-triangular and strictly upper-triangular parts of $\tilde{\lambda}$, and we can treat these cases separately. Actually, these two classes of transformations have very different interpretations: as we shall see shortly, lower-triangular gauge parameters encode the global symmetries of the system, mapping into each other physically inequivalent solutions, while strictly upper-triangular $\tilde{\lambda}$ (and hence $\lambda$ ) generate the proper gauge symmetries discussed in section 3 .

The lower-triangular gauge parameters preserving the diagonal gauge are of the form

$$
\lambda=\frac{1}{\sqrt{k}} \sum_{j=1}^{N} \vec{\epsilon}_{j} \cdot(i \vec{\xi}(z)) e_{j j}+\text { strictly lower triangular },
$$

where $\vec{\xi}(z)$ is a vector of arbitrary holomorphic functions and the remaining strictly lowertriangular part of $\lambda$ is determined by $\vec{\xi}$ through the differential equations (4.4). This 
portion of the resulting gauge parameter depends on the fields: nevertheless, one can still integrate the variation of the charges in (2.10) because its scalar product with the connection vanishes. This means that, even if the gauge parameter is field dependent, one can still use (2.11) to evaluate the asymptotic charges, in complete analogy with what one usually does in the analysis of the global symmetries of Drinfeld-Sokolov gauges (see e.g. $[5,21])$. The choice of a specific solution of the system of equations (4.4), i.e. of the integration constants, is also immaterial, since they do not affect the connection and they drop out of the charges.

Substituting (4.5) into (2.11) taking (3.2) into account, the asymptotic charges eventually read

$$
Q_{\vec{\xi}, \vec{\xi}}=\frac{1}{2 \pi} \oint\left(\vec{\xi} \cdot \partial_{z} \vec{\phi} d z+\vec{\xi} \cdot \partial_{\bar{z}} \vec{\phi} d \bar{z}\right),
$$

while gauge transformations generated by parameters of the form (4.5) induce shifts of the fields that only depend on the entries of the main diagonal:

$$
\delta_{\vec{\xi}, \vec{\xi}} \partial_{z} \vec{\phi}=\partial_{z} \vec{\xi}, \quad \delta_{\vec{\xi}, \vec{\xi}} \partial_{\bar{z}} \vec{\phi}=\partial_{\bar{z}} \vec{\xi}
$$

We now assume that these transformations are generated by the charges as

$$
\delta_{\vec{\xi}, \vec{\xi}} \partial_{z} \vec{\phi}=\left\{Q_{\vec{\xi}, \vec{\xi}}, \partial_{z} \vec{\phi}\right\}
$$

for a proper Poisson bracket defined on the space of fields in the diagonal gauge. ${ }^{8}$

We then expand $\vec{\phi}$ and $\vec{\phi}$ in Laurent modes

$$
i \partial_{z} \phi^{a}=\sum_{m \in \mathbb{Z}} \frac{\alpha_{m}^{a}}{z^{m+1}}, \quad-i \partial_{\bar{z}} \bar{\phi}^{a}=\sum_{m \in \mathbb{Z}} \frac{\bar{\alpha}_{m}^{a}}{\bar{z}^{m+1}} .
$$

Using (4.8) for gauge parameters of the form $\vec{\xi}(z)=\vec{\xi}_{n} z^{n}$ we find for the modes

$$
-i\left\{\alpha_{m}^{a}, \alpha_{n}^{b}\right\}=m \delta^{a b} \delta_{m,-n}, \quad-i\left\{\bar{\alpha}_{m}^{a}, \bar{\alpha}_{n}^{b}\right\}=m \delta^{a b} \delta_{m,-n}, \quad\left\{\alpha_{m}^{a}, \bar{\alpha}_{n}^{b}\right\}=0 .
$$

Note that the zero modes $\vec{\alpha}_{0}$ and $\vec{\alpha}_{0}$ are 'central', in the sense that they Poisson-commute with everything else. In particular, as it stands there are no canonically conjugate variables to $\vec{\alpha}_{0}, \vec{\alpha}_{0}$ in the space of modes. With hindsight, this is not surprising: one cannot expect this procedure to reproduce a bona fide phase space, due to the presence of the residual gauge symmetries discussed in section 3.2 .

To proceed we then propose to slightly extend the space (4.10) by introducing zero modes canonically conjugate to $\vec{\alpha}_{0}$ and $\vec{\alpha}_{0}$ by hand. These can be introduced rather

\footnotetext{
${ }^{8}$ If all constraints were second class, this would be the standard Dirac bracket [28]. In our case, we propose to still apply (4.8) and to then complement the results it gives in such a way to obtain a well defined phase space. This procedure is also supported by the observation that, as discussed in the $\operatorname{sl}(2)$ example in section 2.2, the constraint matrix is invertible almost everywhere, and this suggests that the induced Dirac bracket can be extended to the whole space of fields in the diagonal gauge. This reasoning similarly applies to all $\mathrm{sl}(N)$ cases: as shown in (3.96) of [8], for $\operatorname{sl}(N)$ the constraint matrix indeed has a block-diagonal form as in the sl(2) case. The steps needed to obtain the bracket in (3.99) of [8] are however not as direct as claimed in the latter paper; the non-vanishing block of the constraint matrix is not invertible on the whole constraint surface due to its dependence on phase-space variables.
} 
naturally by promoting the constant mode of $\vec{\phi}$ (which does not appear in the gauge connection $a_{z}$ ) to a dynamical variable, and to declare that it is shifted by acting with a constant gauge parameter $\vec{\xi}$. In other words, we replace (4.7) with its integrated version

$$
\delta_{\vec{\xi}, \vec{\xi}} \vec{\phi}=\vec{\xi}, \quad \delta_{\vec{\xi}, \vec{\xi}} \vec{\phi}=\overrightarrow{\vec{\xi}},
$$

so that the extra zero-modes can be considered as a sort of Stueckelberg fields. From the Laurent expansion of $\vec{\phi}$,

$$
i \vec{\phi}=i \vec{\phi}_{0}+\vec{\alpha}_{0} \log z-\sum_{m \in \mathbb{Z}_{0}} \frac{\vec{\alpha}_{m}}{m z^{m}}
$$

and following the procedure above we find, in addition to (4.10), the zero-mode Poisson brackets

$$
\left\{\alpha_{0}^{a}, \phi_{0}^{b}\right\}=\delta^{a b} .
$$

At this stage we have a concrete proposal for a boundary phase space for any $\operatorname{sl}(N, \mathbb{C})$ Chern-Simons theory satisfying the AdS boundary conditions (2.15) supplemented by the partial gauge fixing of Drinfeld-Sokolov symmetries leading to the diagonal gauge. We know from section 3 that residual gauge symmetries do exist. The goal of the next subsection is to identify the canonical generators of these symmetries on the phase space defined by (4.10) and (4.13). This will allow us to then identify the observables of the theory with the quantities which Poisson commute with these generators, and to verify that they correspond to the charges computed in Drinfeld-Sokolov gauges.

\subsection{Screening charges as generators of residual gauge symmetries}

In the preceding subsection we have formulated Poisson brackets on the space of gauge connections satisfying the diagonal gauge, extended by zero modes $\phi_{0}^{a}$. In this section we will find canonical generators on this phase space which generate the residual gauge symmetries discussed in section 3. Note that in this context the charge formula (2.11) is not expected to be of any help in selecting these generators. For instance the variation $\delta Q \sim$ $\int \operatorname{tr} \lambda \delta a=0$ vanishes for strictly upper triangular $\lambda$ and diagonal $\delta a$, so that the residual symmetries should be associated to constant charges, compatibly with their interpretation as proper gauge symmetries. We will therefore construct directly the functions on the phase space (4.10), (4.13) that generate via (4.8) strictly upper triangular gauge transformations preserving the diagonal gauge.

We have discussed in section 3.2 that we can express the residual gauge transformations of the connection $a_{z}=g^{-1} \partial_{z} g$ as transformations of the type (3.20) on $g(z)$. Infinitesimal transformations can be described by considering $\tilde{\Lambda}_{0}=\mathbb{1}+\epsilon \tilde{\lambda}_{0}$ with a constant strictly upper triangular matrix $\tilde{\lambda}_{0}$ and an infinitesimal parameter $\epsilon$, together with $\Lambda_{-}(z)=\mathbb{1}+\epsilon \lambda_{-}(z)$ such that

$$
\tilde{g}(z)=\left(\mathbb{1}+\epsilon \tilde{\lambda}_{0}\right) g(z)\left(\mathbb{1}+\epsilon \lambda_{-}(z)\right)=g(z)+\epsilon\left(\tilde{\lambda}_{0} g(z)+g(z) \lambda_{-}(z)\right)+\ldots
$$

is lower triangular. One can observe that $\lambda_{-}(z)$ is completely determined in terms of $g$ and $\tilde{\lambda}_{0}$. The infinitesimal action on the gauge connection is then

$$
\delta a_{z}=\partial_{z} \lambda_{-}+\left[a_{z}, \lambda_{-}\right] .
$$


A basis of the infinitesimal transformations is given by the generators

$$
\tilde{\lambda}_{0}^{(i j)}=e_{a b} \quad(a<b) .
$$

Obviously, the Lie algebra that one obtains by considering commutators of such transformations is that of upper triangular matrices, where every element can be generated from the elementary transformations $\tilde{\lambda}_{0}^{(a, a+1)}$. For these elementary transformations one can solve for $\lambda_{-}$, and one obtains

$$
\lambda_{-}^{(a, a+1)}(z)=-h^{-1}(z) e_{a, a+1} h(z)=-e^{-\frac{i}{\sqrt{k}}\left(\vec{\epsilon}_{a}-\vec{\epsilon}_{a+1}\right) \cdot \vec{\phi}(z)} e_{a, a+1} .
$$

Denoting by $\delta_{a}$ the corresponding transformation generated by $\lambda_{-}^{(a, a+1)}$ we find

$$
\delta_{a}\left(i \partial_{z} \vec{\phi}\right)=-\sqrt{k} e^{-\frac{i}{\sqrt{k}} \vec{e}_{a} \cdot \vec{\phi}_{\vec{e}}}, \quad a=1, \ldots, N-1 .
$$

When we look for charges $Q_{a b}$ that generate the transformations associated to $\tilde{\lambda}_{0}^{a b}$, it is enough to find charges $S_{a}=Q_{a, a+1}$ that generate the elementary transformations (4.18), because all other charges $Q_{a b}$ can then be obtained by forming Poisson brackets of the elementary charges. Such charges are given by

$$
S_{a}=\frac{k}{2 \pi} \oint d z e^{-\frac{i}{\sqrt{k}} \vec{e}_{a} \cdot \vec{\phi}(z)} .
$$

Note that $\vec{\phi}$ contains a logarithm, and therefore we have to specify the contour and the branch of the logarithm. As the logarithm only appears together with the zero mode $\alpha_{0}^{a}$, different choices here will only lead to a modified transformation of the zero mode $\phi_{0}^{a}$. Let us now check that the charges generate the desired transformation,

$$
\left\{S_{a}, i \partial_{z} \vec{\phi}\right\}=-\sqrt{k} e^{-\frac{i}{\sqrt{k}} \vec{e}_{a} \cdot \vec{\phi}_{\vec{e}}} .
$$

We first observe that

$$
\left\{e^{-\frac{i}{\sqrt{k}} \vec{e}_{a} \cdot \vec{\phi}(z)}, \vec{\alpha}_{m}\right\}=\frac{i}{\sqrt{k}} \vec{e}_{a} z^{m} e^{-\frac{i}{\sqrt{k}} \vec{e}_{a} \cdot \vec{\phi}(z)}
$$

Then

$$
\begin{aligned}
\left\{S_{a}, i \partial_{z} \vec{\phi}(z)\right\}= & \sum_{m \in \mathbb{Z}} z^{-m-1}\left\{S_{a}, \vec{\alpha}_{m}\right\} \\
= & -\frac{\sqrt{k}}{2 \pi i} \vec{e}_{a} \sum_{m \in \mathbb{Z}} z^{-m-1} \oint_{\left|z^{\prime}\right|=c o n s t .} d z^{\prime} z^{\prime m} e^{-\frac{i}{\sqrt{k}} \vec{e} \cdot \vec{\phi}\left(z^{\prime}\right)} \\
= & -\frac{\sqrt{k}}{2 \pi i} \vec{e}_{a}\left(\oint_{\left|z^{\prime}\right|>|z|} d z^{\prime} \sum_{m<0} z^{-m-1} z^{\prime m} e^{-\frac{i}{\sqrt{k}} \vec{e}_{a} \cdot \vec{\phi}\left(z^{\prime}\right)}\right. \\
& \left.+\oint_{\left|z^{\prime}\right|<|z|} d z^{\prime} \sum_{m \geq 0} z^{-m-1} z^{\prime m} e^{-\frac{i}{\sqrt{k}} \vec{e}_{a} \cdot \vec{\phi}\left(z^{\prime}\right)}\right) \\
= & -\frac{\sqrt{k}}{2 \pi i} \vec{e}_{a}\left(\oint_{\left|z^{\prime}\right|>|z|}-\oint_{\left|z^{\prime}\right|<|z|}\right) d z^{\prime} \frac{1}{z^{\prime}-z} e^{-\frac{i}{\sqrt{k}} \vec{e}_{a} \cdot \vec{\phi}\left(z^{\prime}\right)} \\
= & -\sqrt{k} e^{-\frac{i}{\sqrt{k}} \vec{e}_{a} \cdot \vec{\phi}(z)} \vec{e}_{a} .
\end{aligned}
$$


Note that we have assumed here that $e^{-\frac{i}{\sqrt{k}} \vec{e}_{a} \cdot \vec{\phi}\left(z^{\prime}\right)}$ does not have a cut. As we have seen in section 3.3 this is precisely satisfied for those configurations where the infinitesimal residual gauge transformation is unobstructed.

By a similar computation, one can also determine the transformation of $\vec{\phi}$, including the zero mode, which will depend on the choice of contour in (4.19). This reflects the freedom that we have when extending the transformations from $a_{z}$, labelled by $\partial_{z} \vec{\phi}$, to $\vec{\phi}$, and the precise relation between the transformation of $\vec{\phi}$ and the choice of contour will not be important in the following.

We conclude that we have indeed found the canonical generators of the residual gauge transformations. Note that they vanish on configurations for which the gauge transformations are unobstructed: we observed in section 3.3 that continuous gauge orbits only exist when $e^{-\frac{i}{\sqrt{k}} \vec{e}_{a} \cdot \vec{\phi}}$ has a vanishing residue. Restricting the field space to such configurations can therefore be thought of as implementing the classical screening charges as first-class constraints.

For the physical symmetries of our theory this implies that out of the global symmetries that we found in the previous subsection only those which Poisson commute with the charges $S_{a}$ should be considered as true physical symmetries. As we will see in the following subsection, these symmetries form a classical $W_{N}$ algebra. We will refer to the charges $S_{a}$ as screening charges since, in a sense to be made precise in section 4.4 below, they are a classical limit of the screening charges of the Coulomb gas formalism, see [6].

\subsection{Classical $W_{N}$ algebra}

Having discussed the presence of residual symmetries in the diagonal gauge and having derived the screening charges (4.19) which generate them, we now turn to the construction of gauge-invariant observables which Poisson-commute with the screening charges. In the class of Drinfeld-Sokolov gauges which we reviewed in section 2.2, the DS gauge freedom is completely fixed, and therefore the variables which parameterise the reduced phase space in these gauges should automatically provide us with gauge-invariant observables. It is well-known that these variables are the modes of the $W_{N}$ currents which form a classical $W_{N}$ algebra under Poisson brackets. As a consistency check, we will now verify explicitly that these indeed Poisson-commute with the screening charges (4.19). As an added bonus this analysis will tell us how to relate the diagonal gauge to the DS gauges.

We will focus here on a particularly convenient DS gauge, which is sometimes called the 'U-gauge'. In this case the gauge field takes the form

$$
a_{z}^{U}=J_{1}-\sum_{j=2}^{N}(-\sqrt{k})^{-j} U^{j}(z) e_{1, j} .
$$

It can be shown (see e.g. [29]) that the relation between the $U_{j}$ and the diagonal gauge fields $\phi^{a}$ is given by the classical Miura transformation

$$
M \equiv\left(\partial+\frac{\vec{\epsilon}_{1} \cdot(i \partial \vec{\phi})}{\sqrt{k}}\right) \cdots\left(\partial+\frac{\vec{\epsilon}_{N} \cdot(i \partial \vec{\phi})}{\sqrt{k}}\right)=\partial^{N}-\sum_{j=2}^{N}(-\sqrt{k})^{-j} U_{j} \partial^{N-j}
$$


By comparing the coefficients of $\partial^{N-j}$ in these two ways of writing the differential operator $M$, we obtain expressions for the $W_{N}$ currents $U^{i}$ in terms of the diagonal gauge fields $\partial \vec{\phi}$. For example, for $U^{2}$ and $U^{3}$ one finds, denoting for brevity $\psi_{i}^{\prime} \equiv \vec{\epsilon}_{i} \cdot \partial \vec{\phi}$,

$$
\begin{aligned}
U^{2}= & \sum_{i<j} \psi_{i}^{\prime} \psi_{j}^{\prime}-i \sqrt{k} \sum_{j}(j-1) \psi_{j}^{\prime \prime} \\
= & -\frac{1}{2} \vec{\phi}^{\prime} \cdot \vec{\phi}^{\prime}+i \sqrt{k} \vec{\rho} \cdot \vec{\phi}^{\prime \prime} \\
U^{3}= & -i \sum_{i<j<k} \psi_{i}^{\prime} \psi_{j}^{\prime} \psi_{k}^{\prime}-\sqrt{k} \sum_{i<j}\left((i-1) \partial_{z}\left(\psi_{i}^{\prime} \psi_{j}^{\prime}\right)+(j-i-1) \psi_{i}^{\prime} \psi_{j}^{\prime \prime}\right) \\
& +\frac{i k}{2} \sum_{j}(j-1)(j-2) \psi_{j}^{\prime \prime \prime} .
\end{aligned}
$$

In the second line we have used

$$
\sum_{i<j} \epsilon_{i}^{a} \epsilon_{j}^{b}=-\frac{1}{2} \delta^{a b}, \quad \sum_{j} j \vec{\epsilon}_{j}=-\vec{\rho}
$$

where $\vec{\rho}$ is the Weyl vector. We note that the quantity $U^{2}$ has the form of a free field stress tensor in the presence of a background charge.

As explained at the beginning of this section, the $W_{N}$-currents $U^{i}$ should be completely invariant under DS gauge transformations by construction, which means in particular that they should have vanishing Poisson brackets with the classical screening charges (4.19):

$$
\left\{S_{a}, U^{i}(z)\right\}=\delta_{a} U^{i}(z)=0,
$$

with $\delta_{a}$ defined in (4.18). To check this, we show that the variation $\delta_{a}$ of the left-hand side of (4.28) vanishes. Let us illustrate this for $\delta_{1}$ :

$$
\begin{aligned}
\delta_{1}(L H S)= & {\left[-e^{-\frac{i}{\sqrt{k}} \vec{e}_{1} \cdot \vec{\phi}}\left(\partial+\frac{i \vec{\epsilon}_{2} \cdot \partial \vec{\phi}}{\sqrt{k}}\right)+\left(\partial+\frac{i \vec{\epsilon}_{1} \cdot \partial \vec{\phi}}{\sqrt{k}}\right) e^{-\frac{i}{\sqrt{k}} \vec{e}_{1} \cdot \vec{\phi}}\right] } \\
& \times\left(\partial+\frac{i \vec{\epsilon}_{3} \cdot \partial \vec{\phi}}{\sqrt{k}}\right) \ldots\left(\partial+\frac{i \vec{\epsilon}_{N} \cdot \partial \vec{\phi}}{\sqrt{k}}\right)
\end{aligned}
$$

and one checks using (3.3) that the operator in square brackets vanishes. This classical argument mirrors a similar proof for the quantum screening charges in [6]. Conversely, we demonstrate in appendix B that the screening transformations constitute the most general infinitesimal symmetries of the left hand side of the Miura transformation (4.28).

It is well-known [8] that the currents $U^{i}(z)$ form a classical $W_{N}$ algebra under Poisson brackets, with $U^{2}$ playing the role of the classical Virasoro stress tensor. A special feature of the U-gauge is that the nonlinearities in the right-hand side of the Poisson bracket algebra is at most quadratic in the $U^{i}$. From the analysis above we see that this classical $W_{N}$ algebra arises here as the Poisson-commutant of a set of screening charges (4.19), which is again a classical limit of the Coulomb gas description of the quantum $W_{N}$ algebra. 
As a further check, we compute the classical central charge of this classical $W_{N}$ algebra. Expanding $U^{2}$ in modes,

$$
U^{2}=\sum_{m \in \mathbb{Z}} \frac{L_{m}}{z^{m+2}}
$$

we obtain

$$
L_{m}=\frac{1}{2} \sum_{n \in \mathbb{Z}} \vec{\alpha}_{n} \cdot \vec{\alpha}_{m-n}-\sqrt{k}(m+1) \vec{\rho} \cdot \alpha_{m} .
$$

Using (4.10) one checks that their Poisson brackets give the classical Virasoro algebra

$$
-i\left\{L_{m}, L_{n}\right\}=(m-n) L_{m+n}+\frac{c_{c l}}{12} m\left(m^{2}-1\right) \delta_{m+n, 0}
$$

with classical central charge

$$
c_{c l}=-12 k \vec{\rho} \cdot \vec{\rho}=-N\left(N^{2}-1\right) k .
$$

From (2.3) we see that the classical central charge takes the Brown-Henneaux [30] value $c_{c l}=\frac{3 l}{2 G_{N}}$ when expressed in terms of Newton's constant.

\subsection{Quantization in diagonal gauge}

We now turn to the quantization of the higher-spin gravity theory in the diagonal gauge. At the classical level, we found a set of variables $\phi^{a}$ satisfying free field Poisson brackets. Due to the residual gauge freedom in this gauge, we also found some additional structure: the screening charges $S_{a}$ which generate residual gauge symmetries, and a set of gaugeinvariant observables $U^{i}$ which generate a classical $W_{N}$ algebra. Since our goal is to obtain a quantum theory which is equivalent to the one obtained by quantizing in a DS gauge, we have to preserve this additional structure also at the quantum level.

We start by replacing the Dirac brackets (4.10) with operator commutation relations, $-i\{,\} \rightarrow[$,$] , leading to$

$$
\left[\hat{\alpha}_{m}^{a}, \hat{\alpha}_{n}^{b}\right]=m \delta^{a b} \delta_{m,-n}, \quad\left[\hat{\alpha}_{0}^{a}, \hat{\phi}_{0}^{b}\right]=-i \delta^{a b} .
$$

The associated free field operators, defined through their Laurent expansions as in (4.9), will be denoted by $\hat{\vec{\phi}}(z)$.

Next we set out to construct quantum screening operators $\hat{S}_{a}$ and $W_{N}$ currents $\hat{U}^{i}(z)$. As in (4.28) it will be convenient to package the latter into a quantum Miura operator $\hat{M}$ which should satisfy

$$
\left[\hat{S}_{a}, \hat{M}(z)\right]=0
$$

and such that $\hat{M}$ and $\hat{S}_{a}$ reduce to their classical versions (4.19), (4.28) in the classical large $k$ limit.

Starting with the quantum screening operators, a natural choice is to take them to be the normal-ordered operators

$$
\hat{S}_{a}=\frac{k}{2 \pi} \oint d z: e^{-\frac{i}{\sqrt{k}} \vec{e}_{a} \cdot \hat{\vec{\phi}}}: .
$$


Note that the coefficient of the exponential cannot receive $1 / k$ corrections if we impose the periodicity (3.38) on the fields $\vec{\phi}$ which guarantees that the gauge field has trivial holonomy.

With these screening charges, we learn from the literature on quantum Drinfeld-Sokolov reduction $[6]^{9}$ that the Miura operator $\hat{M}$ is the normal ordered operator version of $M(z)$ in (4.28), while allowing the constant which has the interpretation of a background charge to receive $1 / k$ corrections. ${ }^{10}$ Concretely this means that $\hat{M}$ is of the form

$$
\begin{aligned}
\hat{M}(z) & =:\left(\partial-\frac{\vec{\epsilon}_{1} \cdot(i \partial \hat{\vec{\phi}})}{\tilde{\alpha}_{0}}\right) \cdots\left(\partial-\frac{\vec{\epsilon}_{N} \cdot(i \partial \hat{\vec{\phi}})}{\tilde{\alpha}_{0}}\right): \\
& =\partial^{N}-\sum_{j=2}^{N}\left(\tilde{\alpha}_{0}\right)^{-j} \hat{U}^{j}(z) \partial^{N-j}
\end{aligned}
$$

where the second line defines the quantum $W_{N}$ currents. The parameter $\tilde{\alpha}_{0}$ denotes the quantum corrected background charge, which from (4.28) must behave for large $k$ as

$$
\tilde{\alpha}_{0}=-\sqrt{k}+\mathcal{O}(1) .
$$

One should keep in mind that with our current field normalization, the classical limit is $k \rightarrow-\infty$ while keeping $\hat{\vec{\phi}} / \sqrt{k}$ fixed, so that the operators $\hat{M}(z)$ and $\hat{S}_{a}$ have welldefined limits.

A quantum version of our classical argument in section 4.3, see e.g. eq. (6.46) in [23], shows that (4.39) holds provided we take

$$
\tilde{\alpha}_{0}=-\sqrt{k}+\frac{1}{\sqrt{k}} \text {. }
$$

In the literature (see e.g. [23]) often constants $\alpha_{+}$and $\alpha_{-}$are introduced which are defined by $\alpha_{+} \alpha_{-}=-1, \tilde{\alpha}_{0}=\alpha_{+}+\alpha_{-}$. In our setup these are related to $k$ as

$$
\alpha_{-}=-\sqrt{k}, \quad \alpha_{+}=1 / \sqrt{k} .
$$

It can be shown that the operators $\hat{U}^{j}$ form a quantum $W_{N}$ algebra at central charge

$$
c=(N-1)\left(1-N(N+1) \tilde{\alpha}_{0}^{2}\right) .
$$

It is important to note that the quantum Miura operator (4.41) possesses the symmetry $\alpha_{+} \leftrightarrow \alpha_{-}$, or

$$
\sqrt{k} \leftrightarrow-\frac{1}{\sqrt{k}}
$$

which is not visible at the classical level. An immediate consequence is that the quantum theory possesses a second set of screening operators which commute with the $W_{N}$ currents, namely

$$
\hat{\tilde{S}}_{a}=\frac{k}{2 \pi} \oint d z: e^{i \sqrt{k} \vec{e}_{a} \cdot \vec{\phi}}: .
$$

We will comment on further ramifications of this quantum symmetry in the discussion in section 6 .

\footnotetext{
${ }^{9}$ We follow here the conventions of $[23]$.

${ }^{10}$ This fact was already recognized in the early work [31] in the context of Liouville theory: the background charge in the stress tensor has to be shifted in order for the Liouville potential to remain a weight $(1,1)$ primary in the quantum theory.
} 


\section{Application: quantization of conical solutions}

We argued in section 3.3 that a special role is played by the field configurations $\partial \vec{\phi}$ which lead to a gauge field with trivial holonomy, as these possess a continuous family of residual gauge symmetries. In this section we will study in detail the class of such configurations which have only a simple pole in the origin and at infinity, i.e. where only the zero mode in (4.9) is turned on. These turn out to be precisely the conical surplus solutions studied in [12]. We will also discuss the quantization of these solutions which is straightforward in the current variables. This will allow us to confirm the conjecture of $[12,13]$ that these solutions correspond to a specific subset of degenerate primaries of the $W_{N}$ algebra.

\subsection{Winding sectors and conical solutions}

In this section we focus on classical pure zero mode solutions, where $\vec{\alpha}_{m}=0$ for $m \neq 0$. Before restricting to the solutions satisfying (3.31), we would like to comment on the $L_{0^{-}}$ spectrum of generic pure zero mode solutions. A useful quantity is $L_{0}-\frac{c_{c l}}{24}$, which indicates if the solution lies below the black hole threshold (when it is negative) or above it (when it is positive). From (4.35) we have

$$
L_{0}-\frac{c_{c l}}{24}=\frac{1}{2}\left(\vec{\alpha}_{0}-\sqrt{k} \vec{\rho}\right)^{2} \equiv \frac{1}{2}\left(\vec{\alpha}_{0}^{\mathrm{cyl}}\right)^{2} .
$$

Here, $\vec{\alpha}_{0}^{\text {cyl }}=\vec{\alpha}_{0}-\sqrt{k} \vec{\rho}$ can be interpreted as the zero-mode on the cylinder, which receives a shift due to the background charge as we will see in (5.14) below. For real $\vec{\alpha}_{0}^{\text {cyl }}$, which was assumed in [8], one obtains solutions above the black hole threshold. However, the quantization condition (3.31) leads to imaginary $\vec{\alpha}_{0}$ and $\vec{\alpha}_{0}^{\text {cyl }}$ (recall that $\sqrt{k}$ is imaginary in the regime of interest), and therefore the solutions we are interested in here lie below the black hole threshold.

Now, let us specialize to the zero mode solutions where $\vec{\alpha}_{0}$ is quantized according to $(3.31)$, i.e.

$$
\vec{\alpha}_{0}=-\sqrt{k} \vec{\Lambda}, \vec{\alpha}_{m \neq 0}=0, \quad i \vec{\phi}=-\sqrt{k} \vec{\Lambda} \log z,
$$

where $\vec{\Lambda}$ is an arbitrary element of the weight lattice, and the arbitrary sign has been introduced for later convenience. As discussed at the end of section 3.3, these can be viewed as winding solutions, where $\vec{\Lambda}$ labels the winding sector.

The analysis in section 3.3 also showed that certain finite residual gauge transformations relate different values of the weight vector $\vec{\Lambda}$, which therefore represent the same physical state. From (3.37), these symmetries act on $\vec{\Lambda}$ as shifted Weyl reflections,

$$
\vec{\Lambda} \sim w \cdot \vec{\Lambda}=w(\vec{\Lambda}+\vec{\rho})-\vec{\rho}
$$

where $w(\Lambda)$ is an arbitrary ordinary Weyl reflection. This freedom can be used to take $\vec{\Lambda}+\vec{\rho}$ to be a dominant weight. In terms of the Dynkin labels,

$$
\vec{\Lambda}=\sum_{a=1}^{N-1} \Lambda^{a} \vec{\omega}_{a},
$$


this means that we can take

$$
\Lambda^{a} \geq-1 \quad \text { for } a=1, \ldots, N-1
$$

without loss of generality.

The analysis in section 3.3 showed furthermore that there are extra requirements for the gauge field to have trivial holonomy, which can be summarized in the requirement that the matrix $N_{+}$, which satisfies (3.8), is single-valued. This matrix is lower triangular with 1 's on the diagonal, and working out (3.8) for the winding solutions (5.2) one finds that its components $n_{a b}(a>b)$ should satisfy:

$$
\begin{aligned}
\partial n_{a+1, a} & =-z^{\Lambda^{a}} \\
\partial n_{a+l, a} & =-z^{\Lambda^{a}} n_{a+l, a+1} \quad \text { for } l \geq 2 .
\end{aligned}
$$

The first line re-states (3.25). In order for $N_{+}$to be single-valued, the right hand side of these equations should not have a residue in $z=0$. This will be the case if none of the Dynkin labels $\Lambda^{a}$ equals -1 , and therefore we will further restrict ourselves to the case where

$$
\Lambda^{a} \geq 0 \quad \text { for } a=1, \ldots, N-1 .
$$

The classical $W_{N}$ charges of the winding solutions (5.2), (5.8) can be computed from (4.28), and in particular for the energy $L_{0}$ we get, using (4.35) or (5.1),

$$
L_{0}=k \mathcal{C}_{2}(\vec{\Lambda})
$$

where $\mathcal{C}_{2}(\vec{\Lambda})=\frac{1}{2} \vec{\Lambda} \cdot(\vec{\Lambda}+2 \vec{\rho})$ is the value of the quadratic Casimir of $\operatorname{sl}(N)$. The restriction (5.8) implies that the solutions under consideration have energies (5.9) smaller than or equal to the energy of the AdS vacuum with $\vec{\Lambda}=0$.

We arrived at the winding solutions (5.2), (5.8) by requiring the gauge field to have trivial holonomy. We now relate these solutions to the ones studied in [12], which classified the solutions with trivial holonomy which can be brought into a Drinfeld-Sokolov gauge by a regular gauge transformation. The outcome was a class of solutions which can be viewed as generalized conical defects. Since the analysis of [12] was performed for a cylindrical boundary, we expect that our winding solutions (5.2), (5.8) become precisely the conical solutions of [12] upon conformal mapping to the cylinder. Let us show in more detail that this is indeed the case.

As we already observed in (4.29), the stress tensor for the fields $\vec{\phi}$ contains a background charge term, which implies that $\partial_{z} \vec{\phi}$ fields do not transform as primaries under conformal transformations. Under a finite conformal transformation,

$$
z \rightarrow w=f(z)
$$

the fields transform as ${ }^{11}$

$$
i \partial_{z} \vec{\phi} \rightarrow i \partial_{w} \overrightarrow{\tilde{\phi}}=\left(f^{\prime}\right)^{-1} i \partial_{z} \vec{\phi}+\sqrt{k}\left(f^{\prime}\right)^{-2} f^{\prime \prime} \vec{\rho} .
$$

\footnotetext{
${ }^{11}$ This can be derived from requiring invariance of the connection $A=J_{0} d \rho+$ $\left(e^{\rho} J_{1}+\frac{1}{\sqrt{k}} \sum_{j} \vec{\epsilon}_{j} \cdot\left(i \partial_{z} \vec{\phi}\right)\right) d z$. Indeed, one checks that this leads to (5.10), (5.11) and $\rho \rightarrow \rho-\log f^{\prime}$.
} 
Applying this to the map from the plane to the cylinder,

$$
w=i \log z
$$

we find that a pure zero mode solution maps to

$$
i \partial_{w} \overrightarrow{\tilde{\phi}}=-i \vec{\alpha}_{0}^{\mathrm{cyl}}
$$

with

$$
\vec{\alpha}_{0}^{\mathrm{cyl}}=\vec{\alpha}_{0}-\sqrt{k} \vec{\rho}
$$

In the case of the winding solutions with $\vec{\alpha}_{0}$ given in (5.2) one finds $i \partial_{w} \overrightarrow{\tilde{\phi}}=i \sqrt{k}(\vec{\Lambda}+\vec{\rho})$, and the corresponding gauge connection on the cylinder is

$$
\tilde{a}_{w}=J_{1}+i \sum_{j}\left(\Lambda^{j}-\frac{\sum_{k} \Lambda^{k}}{N}-j+\frac{N+1}{2}\right) e_{j j}
$$

where the $\Lambda^{j}$ are the expansion coefficients in the $\vec{\epsilon}_{j}$ basis: $\vec{\Lambda}=\sum_{j=1}^{N} \Lambda^{j} \vec{\epsilon}_{j}$. These are only defined up to an overall shift, and after appropriately fixing this freedom they can be identified with the number of boxes in the $j$-th row of the Young diagram of the representation with highest weight $\vec{\Lambda}$. The connection (5.15) is gauge-equivalent to that of the conical solutions constructed in [12], in particular it can be diagonalized and then coincides with (5.19) in [12]. The extra condition (5.8) arose in [12] from requiring that the solution can be brought to a Drinfeld-Sokolov gauge by a regular gauge transformation.

To conclude this section, we comment on the fact that the winding solutions (5.2), (5.8) possess some properties which are suggestive of an interpretation as solitons of the theory: their gauge field satisfies a smoothness condition, they have finite energy which scales with the coupling $k$ as in (5.9) and are characterized by a topological winding vector $\vec{\Lambda}$. One should however not push this analogy too far since they have energies below the AdS vacuum, and it is possible to show that they also possess unstable directions [15]. In the quantum theory, the latter property will be reflected in the fact that the corresponding quantum states are primaries of $W_{N}$ representations that are nonunitary in the large central charge limit.

\subsection{Classical symmetries and null vectors}

As we will see below, the winding solutions lead, upon quantization, to degenerate primaries which possess a large number of null descendants. Before getting to this point, we would like to see first how this property manifests itself already at the classical level. As was stressed in $[13,32]$, null vectors are a quantum manifestation of symmetries of the corresponding classical solution. For example, the $\operatorname{sl}(2, \mathbb{R}) \times \operatorname{sl}(2, \mathbb{R})$ symmetry of the global AdS background is directly related to the fact that the corresponding quantum state, the vacuum, has null vectors generated by acting with $L_{-1}$ and $\bar{L}_{-1}$.

We will now see that the winding solutions possess many symmetries. Indeed, as we showed in section 3.3, the winding solutions (5.2) with (5.8) allow for a continuous family of residual gauge transformations. These do not change the physical state and therefore 
generate symmetries of the solution. To find the corresponding infinitesimal symmetry generators, we evaluate the screening charges (4.19) on a configuration $\vec{\phi}$ in the vicinity of a winding solution (5.2) with $\vec{\alpha}_{0}=-\sqrt{k} \vec{\Lambda}$, and expand the result to linear order in the modes. Doing this we obtain

$$
\begin{aligned}
N_{a}(\vec{\Lambda}) & =\left.i k e^{-\frac{i}{\sqrt{k}} \vec{e}_{a} \cdot \vec{\phi}_{0}} \operatorname{Res}_{z \rightarrow 0}\left(z^{\Lambda^{a}} \exp \left(\frac{1}{\sqrt{k}} \sum_{m \neq 0} \frac{\vec{e}_{a} \cdot \vec{\alpha}_{m}}{m z^{m}}\right)\right)\right|_{\text {lin }} \\
& =i \sqrt{k} \frac{\vec{e}_{a} \cdot \vec{\alpha}_{\Lambda^{a}+1}}{\Lambda^{a}+1}
\end{aligned}
$$

We note that the $N_{a}(\vec{\Lambda})$ obtained in this way have $L_{0}$-levels equal to $-\Lambda^{a}-1$, which is negative due to (5.8). The winding solutions also possess symmetry generators at positive levels. To obtain these, we use the fact that we can act with the shifted Weyl reflections (5.3) to give a different representative of the same physical solution. In particular, following [6], we make use of the longest element of the Weyl group, $w_{0}$, which acts on the Dynkin labels as:

$$
\left(w_{0}(\vec{\Lambda})\right)^{a}=-\Lambda^{N-1-a}=-\left(\Lambda^{*}\right)^{a}
$$

(where $\vec{\Lambda}^{*}$ denotes the weight of the representation conjugate to $\vec{\Lambda}$ ). The corresponding shifted Weyl reflection is

$$
w_{0} \cdot \vec{\Lambda}=-\vec{\Lambda}^{*}-2 \vec{\rho} .
$$

The resulting infinitesimal symmetry generator $N_{a}\left(w_{0} \cdot \vec{\Lambda}\right)$ is

$$
N_{a}\left(w_{0} \cdot \vec{\Lambda}\right)=-i \sqrt{k} \frac{\vec{e}_{a} \cdot \vec{\alpha}_{-\Lambda^{N-1-a}-1}}{\Lambda^{N-1-a}+1}
$$

The present method of obtaining symmetry generators by evaluating the screening charges completely mimics the construction of null vectors in degenerate representations of the quantum $W_{N}$ algebra, as we discuss in appendix C. In particular our classical construction (5.20) gives $N-1$ basic symmetry generators at levels $\Lambda^{b}+1, b=1, \ldots, N-1$, which are precisely the levels at which null vectors appear in certain degenerate representations. The result (5.20) can also be derived from an alternative method which was used in [13], where the symmetries of the winding solutions were constructed directly, using the pure gauge form of $a_{z}$ in (3.7).

\subsection{Quantization of conical solutions}

Now let us turn to the quantization of the winding solutions (5.2). Here, by quantization of a classical solution $\vec{\phi}$ we mean the identification of a state in the quantum state space on which the field operator $\hat{\vec{\phi}}$ has the eigenvalue $\vec{\phi}$. In our case the state space consists of Fock spaces built on vacuum states $\left|\vec{\alpha}_{0}, 0\right\rangle$ which satisfy

$$
\hat{\vec{\alpha}}_{0}\left|\vec{\alpha}_{0}, 0\right\rangle=\vec{\alpha}_{0}\left|\vec{\alpha}_{0}, 0\right\rangle, \quad \hat{\vec{\alpha}}_{m}\left|\vec{\alpha}_{0}, 0\right\rangle=0 \text { for } m>0 .
$$

Let us first review some of the properties of the vacuum states $\left|\vec{\alpha}_{0}, 0\right\rangle$ which will be useful below. From (4.41) it follows that they are $W_{N}$ primaries, i.e. they satisfy 
$\hat{U}_{m}^{s}\left|\vec{\alpha}_{0}, 0\right\rangle=0$ for $m>0$. We will denote the corresponding eigenvalues of the zero modes of the $W_{N}$-currents as

$$
\hat{U}_{0}^{s}\left|\vec{\alpha}_{0}, 0\right\rangle \equiv \Delta_{s}\left(\vec{\alpha}_{0}\right)\left|\vec{\alpha}_{0}, 0\right\rangle .
$$

These can be computed by letting the Miura operator (4.41) act on a vacuum state $\left|\vec{\alpha}_{0}, 0\right\rangle$ and inspecting the terms which are singular as $z \rightarrow 0$. This leads to

$$
\left(\partial-\frac{\vec{\epsilon}_{1} \cdot \vec{\alpha}_{0}}{\tilde{\alpha}_{0} z}\right) \ldots\left(\partial-\frac{\vec{\epsilon}_{N} \cdot \vec{\alpha}_{0}}{\tilde{\alpha}_{0} z}\right)=\partial^{N}-\sum_{j=2}^{N}\left(\tilde{\alpha}_{0}\right)^{-j} \Delta_{j}\left(\vec{\alpha}_{0}\right) z^{-j} \partial^{N-j} .
$$

This equation for the $\Delta_{j}\left(\vec{\alpha}_{0}\right)$ can be solved to give [23]

$$
\Delta_{s}\left(\vec{\alpha}_{0}\right)=(-1)^{s-1} \sum_{i_{1}<\ldots<i_{s}} \prod_{j=1}^{s}\left(\vec{\epsilon}_{i_{j}} \cdot \vec{\alpha}_{0}+(s-j) \tilde{\alpha}_{0}\right) .
$$

Now consider a generic classical field configuration $\vec{\phi}(z)$ which is sufficiently regular for large $z$, in the sense that only the modes $\vec{\alpha}_{m}$ for $m \geq 0$ are turned on. According to the definition above, the associated quantum state will typically be a coherent state. However, when only the zero mode $\vec{\alpha}_{0}$ is nonvanishing, the corresponding quantum state is simply the vacuum state $\left|\vec{\alpha}_{0}, 0\right\rangle$. For generic values of $\vec{\alpha}_{0}$, this is a generic $W_{N}$ primary which does not have any null descendants. For the winding solutions (5.2), (5.8) however, the zero mode $\vec{\alpha}_{0}$ takes on the quantized values $\alpha_{-} \vec{\Lambda}$, with $\vec{\Lambda}$ a dominant weight. They correspond to the vacuum states

$$
\left|\alpha_{-} \vec{\Lambda}, 0\right\rangle \text {. }
$$

The corresponding $W_{N}$ charges are, from (5.24),

$$
\Delta_{s}\left(\alpha_{-} \vec{\Lambda}\right)=(-1)^{s-1} \sum_{i_{1}<\ldots<i_{s}} \prod_{j=1}^{s}\left(\alpha_{-} \vec{\epsilon}_{i_{j}} \cdot \vec{\Lambda}+(s-j) \tilde{\alpha}_{0}\right),
$$

in particular, the conformal weights read

$$
\Delta_{2}\left(\alpha_{-} \vec{\Lambda}\right)=\frac{\alpha_{-}}{2} \vec{\Lambda} \cdot\left(\alpha_{-} \vec{\Lambda}+2 \tilde{\alpha}_{0} \vec{\rho}\right) .
$$

From these observations we can immediately identify the winding solutions with the primaries of a subset of completely degenerate representations of the $W_{N}$ algebra. Here, the term completely degenerate refers to a representation which contains at least $N-1$ singular vectors (i.e. primary null vectors). Completely degenerate $W_{N}$ representations are labelled by two $\operatorname{sl}(N)$ weight vectors $\left(\vec{\Lambda}^{\prime}, \vec{\Lambda}\right)$, and in the free field state space the corresponding $W_{N}$ primaries are [23]

$$
\left|\alpha_{+} \vec{\Lambda}^{\prime}+\alpha_{-} \vec{\Lambda}, 0\right\rangle
$$

Therefore the conical/winding solutions $(5.25)$ correspond precisely to the $(0, \vec{\Lambda})$ degenerate primaries. The fact that the representations built on these primaries are completely degenerate can be seen from the explicit construction of the null vectors [6], which we review in appendix $\mathrm{C}$. The operators which create the null vectors reduce in the classical limit to the symmetry generators we found in (5.20). 
These considerations give a quantum check, to all orders in an expansion in $1 / k$, of the conjecture $[12,13]$ that the conical/winding solutions $(5.2)$ correspond to the $(0, \vec{\Lambda})$ degenerate primaries. Previous checks of the conjecture include matching all the $W_{N}$ charges (5.26) in the classical, large $k$ limit [14] and matching the first quantum $1 / k$ correction for $N=2$ [15]. We note that in the current variables, the full effect of quantization was simply encoded in the fact that the quantum background charge is shifted from its classical value according to (4.44). ${ }^{12}$

\section{Discussion and outlook}

In this work we have formulated the $\operatorname{sl}(N, \mathbb{C})$ higher-spin theories in the diagonal gauge, and shown that this leads to a classical version of the Coulomb gas formalism. In particular, we saw that the screening charges emerge naturally as generators of residual gauge symmetries. As an example of the power of this formulation, we completed the proof that the soliton-like winding solutions in the classical theory represent the $(0, \vec{\Lambda})$ degenerate primaries in the dual CFT. Before discussing some open questions, we now would like to comment further on the implications of the $\mathbb{Z}_{2}$ symmetry which we encountered in (4.47).

Quantum duality and the matter spectrum. In section 4.4 we showed that the observables in the quantum theory are invariant under the following symmetry:

$$
\alpha_{+} \leftrightarrow \alpha_{-} \quad \text { or } \quad \sqrt{k} \leftrightarrow-\frac{1}{\sqrt{k}}
$$

This is a symmetry of the quantum $W_{N}$ algebra, since it leaves the Miura operator (4.41) invariant, which is not visible in the classical large $k$ limit [6]. It can be seen as a part of the triality symmetry of the $\mathcal{W}_{\infty}[N]$ algebra which survives the truncation to $W_{N}$ [11].

It is interesting to explore the consequences of the following two natural assumptions:

- (6.1) is a symmetry of the full quantum theory, in particular it is also a symmetry of the spectrum.

- the spectrum contains the soliton-like winding states (5.2) with $\vec{\alpha}_{0}=\alpha_{-} \vec{\Lambda}$.

The $\mathbb{Z}_{2}$ symmetry then predicts that the theory should also contain the states with $\vec{\alpha}_{0}=\alpha_{+} \vec{\Lambda}$, which are the primaries of the degenerate representation $(\vec{\Lambda}, 0)$. These do not correspond to regular classical configurations of the higher-spin gauge field, since they do not satisfy the condition (3.31) and therefore the gauge field contains a singularity corresponding to an external point particle source in the form of a Wilson line [33, 34]. The inclusion of these states therefore requires adding matter particles to the massless higher-spin theory. The energy of these matter states behaves at large $k$ as

$$
\Delta_{2}\left(\alpha_{+} \vec{\Lambda}^{\prime}\right) \approx-\vec{\Lambda}^{\prime} \cdot \vec{\rho}+\mathcal{O}\left(k^{-1}\right) .
$$

\footnotetext{
${ }^{12}$ Note that in our setup, this should not be viewed as a quantum correction to the coupling constant $k$, since $k$ plays a double role: it determines both the size of the winding mode lattice (3.31) and the value of the background charge, and the only the latter quantity receives quantum corrections according to (4.44).
} 
The lightest of these excitations comes from taking $\vec{\Lambda}=\vec{\epsilon}_{1}$ to be the highest weight of the $\mathbf{N}$-dimensional representation and has $\Delta_{2}=\bar{\Delta}_{2}=\frac{1-N}{2}$. This corresponds to a scalar particle with mass

$$
M^{2} l^{2}=4 \Delta_{2}\left(\Delta_{2}-1\right)=N^{2}-1 .
$$

This is precisely the mass of the scalar field coupled to $h s[\lambda]$ massless higher-spin fields in the theory of Prokushkin and Vasiliev [2], in the $\lambda \rightarrow N$ limit. One can also show that the full set of degenerate primaries $\left(\vec{\Lambda}^{\prime}, \vec{\Lambda}\right)$ arises from considering scalar field excitations in soliton backgrounds [13]. Therefore the assumptions above essentially predict that the matter spectrum the theory agrees with that of the Prokushkin-Vasiliev theory. It would be interesting to generalize these results for the $\operatorname{SL}(N, \mathbb{C})$ theory to the $h s[\lambda]$ setting [35].

On a related note, one observes that the $\mathbb{Z}_{2}$ symmetry (6.1) is somewhat reminiscent of T-duality, since it inverts $\sqrt{k}$ which sets the scale for the winding lattice, $\sqrt{k} W$, with $W$ the weight lattice. It is in general not quite T-duality however, since that would also replace the weight lattice by its dual, the root lattice $R$, divided by two. Only for pure gravity, $N=2$, this is ordinary T-duality, since in that case we have that $R / 2=W$. The fact that the degenerate representations of the Virasoro algebra can be viewed as momentum and winding modes of a compact free boson was already pointed out in [36] (see also the early work [37]). It is however amusing to note that, for general $N$, the $\mathbb{Z}_{2}$ symmetry can be viewed as a T-duality in the theory of $N-1$ compact bosons in the presence of a B-field, as we show in appendix D. However, the physical interpretation of such a B-field is not clear to us at present.

Outlook. We conclude by pointing out some open questions and possible applications of our work.

- We have dealt with the residual gauge symmetries of the diagonal gauge in a somewhat ad hoc manner, which led us essentially to the original version of the Coulomb gas formalism in [6]. It would be interesting to see if a more rigorous treatment using BRST methods would lead similarly to the BRST formulation of the Coulomb gas $[36,38]$. It might be hoped that the connection to bulk higher-spin symmetry would give a natural explanation for some of the less obvious computational rules of the Coulomb gas formalism.

- We have mainly focused here on classical solutions with simple poles or 'centers' only in the origin and at infinity, which encode two-point functions of heavy operators in the dual CFT. It would be interesting to explore if the diagonal gauge facilitates the construction of multi-centered solutions which would encode $n$-point correlators of heavy operators.

- It will also be interesting to include chemical potentials in the diagonal gauge in order to discuss higher-spin black holes [20].

- The free field parameterisation discussed in this work may also prove useful in the context of the AGT correspondence [39, 40]. In that context, as explained in [41], a 
particular compactification of the $a_{N-1}(2,0)$ theory gives rise to an $\operatorname{sl}(N, \mathbb{C})$ ChernSimons theory with Nahm pole boundary conditions which implement the DrinfeldSokolov constraint (2.15).

\section{Acknowledgments}

We thank Laszlo Fehér, Olaf Krüger and Tomáš Procházka for useful discussions. A.C. acknowledges the support of the Université libre de Bruxelles, where part of this work has been performed. His work has been partially supported by the ERC Advanced Grant "HighSpin-Grav", by FNRS-Belgium (convention FRFC PDR T.1025.14 and convention IISN 4.4503.15) and by the NCCR SwissMAP, funded by the Swiss National Science Foundation. The research of J.R. was supported by the Grant Agency of the Czech Republic under the grant 17-22899S, and by ESIF and MEYS (Project CoGraDS - CZ.02.1.01/0.0/0.0/15 003/0000437). This collaboration was supported by the bilateral collaboration grant WBI 14-1 between the Fédération Wallonie-Bruxelles and the Academy of Sciences of the Czech Republic. A.C. and J.R. wish to thank U. Vienna for hospitality during the completion of this work.

\section{A Gravity subsector}

Here we review how the theory (2.1) includes Euclidean $\mathrm{AdS}_{3}$ gravity, and compute the relation between $k$ and Newton's constant. For this we must choose a spin-2 subsector, which we take to correspond to the principal embedding of $\operatorname{sl}(2, \mathbb{C})$ into $\operatorname{sl}(N, \mathbb{C})$. With this choice, it is convenient for the present purpose to take the $\operatorname{sl}(2, \mathbb{C})$ generators to be the $N$-dimensional $s u(2)$ representation matrices $K_{i}(i=1,2,3)$ satisfying

$$
\begin{aligned}
{\left[K_{i}, K_{j}\right] } & =-\epsilon_{i j k} \delta^{k l} K_{l}, \\
\operatorname{tr}_{\mathbf{N}} K_{i} K_{j} & =-\frac{N\left(N^{2}-1\right)}{12} \delta_{i j}, \\
K_{i}^{\dagger}= & =-K_{i},
\end{aligned}
$$

with $\epsilon_{123}=1$ (for example, for $N=2$ we can take $K_{j}=i \sigma_{j} / 2$ ). We restrict $A$ to lie in this $\operatorname{sl}(2, \mathbb{C})$ subalgebra and decompose

$$
A=\left(\omega^{j}+\frac{i}{l} e^{j}\right) K_{j}
$$

with $\omega^{j}$ and $e^{j}$ real one-forms. The $s u(2)$-valued part $\omega$ plays the role of the spin connection, while $e$ is the dreibein. Indeed, one shows that the Euclidean action (2.1) can, up to a boundary term, be written as

$$
\begin{aligned}
I_{E} & =\frac{k}{l \pi} \int \operatorname{tr}_{\mathbf{N}}\left(e \wedge \mathcal{R}-\frac{1}{3 l^{2}} e \wedge e \wedge e\right) \\
& =-\frac{N\left(N^{2}-1\right) k}{24 \pi l} \int d^{3} x \operatorname{det} e\left(R+\frac{2}{l^{2}}\right),
\end{aligned}
$$


where $\mathcal{R}=d \omega+\omega \wedge \omega$ is the curvature two-form, with $\mathcal{R}_{i}=\frac{1}{2} \epsilon_{i j k} \mathcal{R}^{j k}$. Choosing the orientation such that $\operatorname{det} e>0$, we can identify $\operatorname{det} e=\sqrt{g}$ and the action (A.6) takes the Einstein-Hilbert form. Therefore the theory (2.1) contains a spin-2 gravity sector with $k$ related to the AdS radius and Newton's constant as in (2.3).

\section{B Infinitesimal symmetries of Miura transform}

We will investigate here the infinitesimal symmetries of the classical Miura transformation (4.28). Under an infinitesimal transformation

$$
i \partial \vec{\phi} \rightarrow i \partial \vec{\phi}+\vec{\eta},
$$

the left hand side of the Miura transformation (4.28) changes as

$$
\delta M=-\frac{1}{\sqrt{k}} \sum_{a=1}^{N-1}\left(\prod_{j=1}^{a-1}\left(\partial+\frac{\vec{\epsilon}_{j} \cdot(i \partial \vec{\phi})}{\sqrt{k}}\right)\right)\left(\partial \eta_{a}+\eta_{a} \frac{\vec{e}_{a} \cdot(i \partial \vec{\phi})}{\sqrt{k}}\right)\left(\prod_{j=a+2}^{N}\left(\partial+\frac{\vec{\epsilon}_{j} \cdot(i \partial \vec{\phi})}{\sqrt{k}}\right)\right),
$$

where we expanded $\vec{\eta}=\sum_{a=1}^{N-1} \eta_{a} \vec{e}_{a}$. The new combination that appears in the sum above will be denoted as

$$
\eta_{a, 1}:=\partial \eta_{a}+\eta_{a} \frac{\vec{e}_{a} \cdot(i \partial \vec{\phi})}{\sqrt{k}}
$$

In the expression for $\delta M$ above we now move all factors containing a derivative to the right, and we obtain

$$
\begin{aligned}
(-\sqrt{k}) \delta M= & \left(\eta_{1,1}+\cdots+\eta_{N-1,1}\right) \prod_{j=3}^{N}\left(\partial+\frac{\vec{\epsilon}_{j} \cdot(i \partial \vec{\phi})}{\sqrt{k}}\right) \\
& +\left(\eta_{2,2}+\cdots+\eta_{N-1,2}\right) \prod_{j=4}^{N}\left(\partial+\frac{\vec{\epsilon}_{j} \cdot(i \partial \vec{\phi})}{\sqrt{k}}\right) \\
& \vdots \\
& +\left(\eta_{N-2, N-2}+\eta_{N-1, N-2}\right)\left(\partial+\frac{\vec{\epsilon}_{N} \cdot(i \partial \vec{\phi})}{\sqrt{k}}\right) \\
& +\eta_{N-1, N-1},
\end{aligned}
$$

where we recursively introduced the combinations

$$
\eta_{a, b}:=\left(\partial+\frac{i}{\sqrt{k}}\left(\vec{\epsilon}_{a+1-b}-\vec{\epsilon}_{a+1}\right) \cdot(\partial \vec{\phi})\right) \sum_{l=a}^{N-1} \eta_{l, b-1} .
$$

Note that this is consistent with (B.3) when we identify $\eta_{a, 0}:=\eta_{a}-\eta_{a+1}$ (setting $\eta_{N}=0$ ). Requiring that the left hand side of the Miura transformation does not change, $\delta M=0$, then leads us to the condition

$$
\sum_{a=b}^{N-1} \eta_{a, b}=0 \quad, b=1, \ldots, N-1 .
$$


Any $\eta_{a}$ that satisfies (B.6) with (B.5) defines an infinitesimal symmetry of the Miura transformation. We now show that such a transformation can always be obtained by a residual gauge transformation. For that purpose we define an upper triangular matrix $\tilde{\lambda}$ by

$$
\tilde{\lambda}_{i, j}=e^{\frac{i}{\sqrt{k}}\left(\vec{\epsilon}_{i}-\vec{\epsilon}_{j}\right) \cdot \vec{\phi}} \sum_{a=j-1}^{N-1} \eta_{a, j-i-1} \quad \text { for } j>i .
$$

We claim that this matrix satisfies equation (4.4), and hence belongs to a transformation that preserves the diagonal gauge. To show this we note that the equations (B.6) satisfied by the $\eta$ 's allow us to formally extend (B.7) to $i=0$ by setting $\tilde{\lambda}_{0, j}=0$. More trivially, the identification (B.7) is also consistent with setting $\tilde{\lambda}_{i, N+1}=0$. Having said this, we can evaluate the right hand side of (4.4) for any $1 \leq i<j \leq N$ using (B.7) and we obtain:

$$
\begin{aligned}
e^{-\frac{i}{\sqrt{k}} \vec{e}_{i-1} \cdot \vec{\phi}} \tilde{\lambda}_{i-1, j}-e^{-\frac{i}{\sqrt{k}} \vec{e}_{j} \cdot \vec{\phi}} \tilde{\lambda}_{i, j+1}= & e^{-\frac{i}{\sqrt{k}}\left(\vec{\epsilon}_{i-1}-\vec{\epsilon}_{i}\right) \cdot \vec{\phi}} e^{\frac{i}{\sqrt{k}}\left(\vec{\epsilon}_{i-1}-\vec{\epsilon}_{j}\right) \cdot \vec{\phi}} \sum_{a=j-1}^{N-1} \eta_{a, j-i} \\
& -e^{-\frac{i}{\sqrt{k}}\left(\vec{\epsilon}_{j}-\vec{\epsilon}_{j+1}\right) \cdot \vec{\phi}} e^{\frac{i}{\sqrt{k}}\left(\vec{\epsilon}_{i}-\vec{\epsilon}_{j+1}\right) \cdot \vec{\phi}} \sum_{a=j}^{N-1} \eta_{a, j-i} \\
= & e^{\frac{i}{\sqrt{k}}\left(\vec{\epsilon}_{i}-\vec{\epsilon}_{j}\right) \cdot \vec{\phi}} \eta_{j-1, j-i} .
\end{aligned}
$$

On the other hand, we plug (B.7) into the left hand side of (4.4), and we find

$$
\begin{aligned}
\partial \tilde{\lambda}_{i, j} & =e^{\frac{i}{\sqrt{k}}\left(\vec{\epsilon}_{i}-\vec{\epsilon}_{j}\right) \cdot \vec{\phi}}\left(\partial+\frac{i}{\sqrt{k}}\left(\vec{\epsilon}_{i}-\vec{\epsilon}_{j}\right) \cdot(\partial \vec{\phi})\right) \sum_{a=j-1}^{N-1} \eta_{a, j-i-1} \\
& =e^{\frac{i}{\sqrt{k}}\left(\vec{\epsilon}_{i}-\vec{\epsilon}_{j}\right) \cdot \vec{\phi}} \eta_{j-1, j-i},
\end{aligned}
$$

which agrees with (B.9). Hence, the upper triangular matrix $\tilde{\lambda}$ defines an allowed transformation. We can quickly check that its effect on $i \partial \vec{\phi}$ is just a shift by $\vec{\eta}$ by observing that

$$
\lambda_{i, i+1}=e^{-\frac{i}{\sqrt{k}}\left(\vec{\epsilon}_{i}-\vec{\epsilon}_{i+1}\right) \cdot \vec{\phi}} \tilde{\lambda}_{i, i+1}=\eta_{i}
$$

We conclude that the most general infinitesimal symmetry of the Miura transformation corresponds to a residual gauge symmetry in the diagonal gauge.

\section{Null vectors and their classical limits}

It is instructive to see how our construction of classical null vectors in section 5.2 gets corrected in the quantum theory [6]. First we observe that the quantum-corrected version of the finite screening transformations (3.29) reads

$$
\vec{\alpha}_{0} \sim w \cdot \vec{\alpha}_{0} \equiv w\left(\vec{\alpha}_{0}+\tilde{\alpha}_{0} \vec{\rho}\right)-\tilde{\alpha}_{0} \vec{\rho}
$$

for any Weyl reflection $w$. It can be shown [23] that these identifications leave the $W_{N}$ charges invariant. To construct the null descendants of our winding states $\left|\alpha_{-} \vec{\Lambda}, 0\right\rangle$ we apply the screening charges $\hat{S}_{a}$ to the $W_{N}$ primaries

$$
\left|w_{0} \cdot\left(\alpha_{-} \vec{\Lambda}\right)+\alpha_{+} \vec{e}_{a}, 0\right\rangle=: e^{i\left(w_{0} \cdot\left(\alpha_{-} \vec{\Lambda}\right)+\alpha_{+} \vec{e}_{a}\right) \cdot \hat{\vec{\phi}}(0)}:|0,0\rangle
$$


with $w_{0}$ the Weyl reflection defined in (5.18). Note that the shift by $\alpha_{+} \vec{e}_{a}$ vanishes in the classical large $k$ limit and was therefore not visible in the classical discussion in section 5.2. Using the free field OPE of $\hat{\vec{\phi}}(z)$ we find

$$
\begin{array}{r}
\hat{S}_{a}\left|w_{0} \cdot\left(\alpha_{-} \vec{\Lambda}\right)+\alpha_{+} \vec{e}_{a}, 0\right\rangle= \\
\frac{i k}{\left(\Lambda^{N-1-a}+1\right) !}\left(\partial_{z}^{\Lambda^{N-1-a}+1}: e^{-i \alpha_{+} \vec{e}_{a} \cdot \hat{\vec{\phi}}(z)} e^{i\left(w_{0} \cdot\left(\alpha_{-} \vec{\Lambda}\right)+\alpha_{+} \vec{e}_{a}\right) \cdot \hat{\vec{\phi}}(0)}:\right)_{\mid z=0}|0,0\rangle \\
\sim\left(-\frac{i \sqrt{k}}{\Lambda^{N-1-a}+1} \vec{e}_{a} \cdot \hat{\vec{\alpha}}_{-\left(\Lambda^{N-1-a}+1\right)}+\mathcal{O}(1)\right)\left|w_{0} \cdot\left(\alpha_{-} \vec{\Lambda}\right), 0\right\rangle .
\end{array}
$$

This state is a $W_{N}$ primary by construction and, from the right-hand side, is also a descendent of $\left|w_{0} \cdot\left(\alpha_{-} \vec{\Lambda}\right), 0\right\rangle \sim\left|\alpha_{-} \vec{\Lambda}, 0\right\rangle$ at level $\Lambda^{N-1-a}+1$. In the last line we displayed the leading large $k$ part, which agrees with the classical result (5.20).

\section{All degenerate primaries as momentum-winding states}

We will show here that all $W_{N}$ degenerate primaries can be described as pure momentumwinding states in a version of the Coulomb gas formalism, where the free fields take values on a certain torus and a constant $B$-field is turned on. The only effect of the latter is to modify the winding lattice, see e.g. [42].

Recall that, in the free field formalism with $N-1$ free fields, a general degenerate $W_{N}$-primary corresponds to a state $\left|\vec{\alpha}_{0}, 0\right\rangle$, where the zero-mode eigenvalue is

$$
\vec{\alpha}_{0}=\alpha_{+} \vec{\Lambda}^{\prime}+\alpha_{-} \vec{\Lambda} \text {. }
$$

Here, $\vec{\Lambda}$ and $\vec{\Lambda}^{\prime}$ are dominant weights of $\operatorname{sl}(N)$, and $\alpha_{ \pm}$are related to the background charge as in (4.45).

We want to reproduce the zero mode quantization condition (D.1) as arising from momentum and winding number quantization in a theory where the free fields $\vec{\phi}$ are periodic, taking values in some torus, in the presence of a constant $B$-field. If the periods are

$$
\vec{\phi} \sim \vec{\phi}+2 \pi n^{a} \vec{l}_{a} \quad \text { (summation over } a=1, \ldots, N-1 \text { ) }
$$

where $n^{a} \in \mathbb{Z},\left\{l_{a}\right\}_{a}$ form a lattice basis, the left-moving zero mode is quantized as (see e.g. [42], eq. (10.49), with $\left.\alpha^{\prime}=2\right)$ ):

$$
\vec{\alpha}_{0}=m_{a} \vec{l}^{* a}-\frac{1}{2} w^{a}\left(g_{a b}+b_{a b}\right) \vec{l}^{* b}
$$

where $m_{a}$ and $w^{a}$ are integer momentum and winding numbers, $\left\{l^{* a}\right\}_{a}$ are dual basis vectors satisfying $l_{a} \cdot l^{* b}=\delta_{a}^{b}, g_{a b}=l_{a} \cdot l_{b}$, and $b_{a b}$ is the constant $B$-field.

We now take the period lattice to be the root lattice divided by $\alpha_{+}$, with basis vectors given by

$$
\overrightarrow{l_{a}}=\frac{1}{\alpha_{+}} \vec{e}_{a}
$$

so that the dual lattice is the weight lattice multiplied by $\alpha_{+}$, with dual basis vectors

$$
\vec{l}^{* a}=\alpha_{+} \vec{\omega}_{a} .
$$


The metric $g_{i j}$ is proportional to the $\operatorname{sl}(N)$ Cartan matrix,

$$
g_{i j}=\frac{1}{\alpha_{+}^{2}} C_{i j}=\frac{1}{\alpha_{+}^{2}}\left(\begin{array}{cccc}
2 & -1 & 0 & \ldots \\
-1 & 2 & -1 & \ldots \\
0 & -1 & 2 & -1 \\
& & & \ldots
\end{array}\right)
$$

Finally, for the $B$-field we take

$$
b_{i j}=\frac{1}{\alpha_{+}^{2}}\left(\begin{array}{cccc}
0 & 1 & 0 & \ldots \\
-1 & 0 & 1 & \ldots \\
0 & -1 & 0 \\
& & & \ldots
\end{array}\right)
$$

Plugging this all into (D.3), we obtain indeed (D.1) with

$$
\begin{aligned}
\vec{\Lambda} & =m_{a} \vec{\omega}_{a} \\
\vec{\Lambda}^{\prime} & =w^{a} \vec{\epsilon}_{a}
\end{aligned}
$$

where the $\vec{\epsilon}_{a}$ are, as before, the first $N-1$ weights of the fundamental representation,

$$
\vec{\epsilon}_{a}=\vec{\omega}_{a}-\vec{\omega}_{a-1}
$$

The $\vec{\epsilon}_{a}$ also form a basis of the weight lattice; this follows from the fact that the transformation (D.10) involves integer coefficients and so does its inverse

$$
\vec{\omega}_{a}=\sum_{b=1}^{a} \vec{\epsilon}_{b}
$$

Hence, by choosing the momentum and winding numbers such that $\vec{\Lambda}$ and $\vec{\Lambda}^{\prime}$ are dominant weights, all the degenerate representations (D.1) are reproduced as momentum-winding states in this theory. We note that for $N=2$, where the $B$-field is absent, this reduces to the observation made in [36].

Open Access. This article is distributed under the terms of the Creative Commons Attribution License (CC-BY 4.0), which permits any use, distribution and reproduction in any medium, provided the original author(s) and source are credited.

\section{References}

[1] M.R. Gaberdiel and R. Gopakumar, An AdS 3 Dual for Minimal Model CFTs, Phys. Rev. D 83 (2011) 066007 [arXiv: 1011.2986] [INSPIRE].

[2] S.F. Prokushkin and M.A. Vasiliev, Higher spin gauge interactions for massive matter fields in 3-D AdS space-time, Nucl. Phys. B 545 (1999) 385 [hep-th/9806236] [InSPIRE].

[3] V.G. Drinfeld and V.V. Sokolov, Lie algebras and equations of Korteweg-de Vries type, J. Sov. Math. 30 (1984) 1975 [InSPIRE]. 
[4] M. Henneaux and S.-J. Rey, Nonlinear $W_{\infty}$ as asymptotic symmetry of three-dimensional higher spin anti-de Sitter gravity, JHEP 12 (2010) 007 [arXiv: 1008.4579] [INSPIRE].

[5] A. Campoleoni, S. Fredenhagen, S. Pfenninger and S. Theisen, Asymptotic symmetries of three-dimensional gravity coupled to higher-spin fields, JHEP 11 (2010) 007 [arXiv: 1008.4744] [INSPIRE].

[6] V.A. Fateev and S.L. Lukyanov, The Models of Two-Dimensional Conformal Quantum Field Theory with $Z_{n}$ Symmetry, Int. J. Mod. Phys. A 3 (1988) 507 [inSPIRE].

[7] S.L. Lukyanov and V.A. Fateev, Additional symmetries and exactly soluble models in two-dimensional conformal field theory, Chur, Switzerland: Harwood (1990), Soviet Scientific Reviews A, Physics: 15.2.

[8] J. Balog, L. Feher, L. O'Raifeartaigh, P. Forgacs and A. Wipf, Toda Theory and W Algebra From a Gauged WZNW Point of View, Annals Phys. 203 (1990) 76 [INSPIRE].

[9] H. Afshar, S. Detournay, D. Grumiller, W. Merbis, A. Perez, D. Tempo et al., Soft Heisenberg hair on black holes in three dimensions, Phys. Rev. D 93 (2016) 101503 [arXiv: 1603.04824] [INSPIRE].

[10] D. Grumiller, A. Perez, S. Prohazka, D. Tempo and R. Troncoso, Higher Spin Black Holes with Soft Hair, JHEP 10 (2016) 119 [arXiv:1607.05360] [INSPIRE].

[11] M.R. Gaberdiel and R. Gopakumar, Triality in Minimal Model Holography, JHEP 07 (2012) 127 [arXiv: 1205.2472] [INSPIRE].

[12] A. Castro, R. Gopakumar, M. Gutperle and J. Raeymaekers, Conical Defects in Higher Spin Theories, JHEP 02 (2012) 096 [arXiv:1111.3381] [INSPIRE].

[13] E. Perlmutter, T. Prochazka and J. Raeymaekers, The semiclassical limit of $W_{N}$ CFTs and Vasiliev theory, JHEP 05 (2013) 007 [arXiv: 1210.8452] [INSPIRE].

[14] A. Campoleoni and S. Fredenhagen, On the higher-spin charges of conical defects, Phys. Lett. B 726 (2013) 387 [arXiv:1307.3745] [INSPIRE].

[15] J. Raeymaekers, Quantization of conical spaces in 3D gravity, JHEP 03 (2015) 060 [arXiv: 1412.0278] [INSPIRE].

[16] S. Datta, M.R. Gaberdiel, W. Li and C. Peng, Twisted sectors from plane partitions, JHEP 09 (2016) 138 [arXiv:1606.07070] [INSPIRE].

[17] M.P. Blencowe, A Consistent Interacting Massless Higher Spin Field Theory in D = (2+1), Class. Quant. Grav. 6 (1989) 443 [inSPIRE].

[18] E. Witten, Quantization of Chern-Simons Gauge Theory With Complex Gauge Group, Commun. Math. Phys. 137 (1991) 29 [inSPIRE].

[19] C. Bunster, M. Henneaux, A. Perez, D. Tempo and R. Troncoso, Generalized Black Holes in Three-dimensional Spacetime, JHEP 05 (2014) 031 [arXiv:1404.3305] [INSPIRE].

[20] M. Gutperle and P. Kraus, Higher Spin Black Holes, JHEP 05 (2011) 022 [arXiv: 1103.4304] [INSPIRE].

[21] M. Bañados, Three-dimensional quantum geometry and black holes, AIP Conf. Proc. 484 (1999) 147 [hep-th/9901148] [INSPIRE].

[22] M. Bañados, Global charges in Chern-Simons field theory and the (2+1) black hole, Phys. Rev. D 52 (1996) 5816 [hep-th/9405171] [INSPIRE]. 
[23] P. Bouwknegt and K. Schoutens, W symmetry in conformal field theory, Phys. Rept. 223 (1993) 183 [hep-th/9210010] [INSPIRE].

[24] R.A. Horn and C.R. Johnson, Matrix Analysis, Cambridge University Press (2012).

[25] V.N. Gribov, Quantization of Nonabelian Gauge Theories, Nucl. Phys. B 139 (1978) 1 [INSPIRE].

[26] E. Witten, Coadjoint Orbits of the Virasoro Group, Commun. Math. Phys. 114 (1988) 1 [INSPIRE].

[27] Z. Bajnok and D. Nogradi, Geometry of W algebras from the affine Lie algebra point of view, J. Phys. A 34 (2001) 4811 [hep-th/0012190] [INSPIRE].

[28] J.D. Brown and M. Henneaux, On the Poisson Brackets of Differentiable Generators in Classical Field Theory, J. Math. Phys. 27 (1986) 489 [inSPIRE].

[29] M. Bershadsky and H. Ooguri, Hidden SL(n) Symmetry in Conformal Field Theories, Commun. Math. Phys. 126 (1989) 49 [INSPIRE].

[30] J.D. Brown and M. Henneaux, Central Charges in the Canonical Realization of Asymptotic Symmetries: An Example from Three-Dimensional Gravity, Commun. Math. Phys. 104 (1986) 207 [INSPIRE].

[31] T.L. Curtright and C.B. Thorn, Conformally invariant quantization of the Liouville theory, Phys. Rev. Lett. 48 (1982) 1309 [Erratum ibid. 48 (1982) 1768] [INSPIRE].

[32] A. Castro, T. Hartman and A. Maloney, The Gravitational Exclusion Principle and Null States in Anti-de Sitter Space, Class. Quant. Grav. 28 (2011) 195012 [arXiv:1107.5098] [INSPIRE].

[33] E. Witten, Topology changing amplitudes in $(2+1)$-dimensional gravity, Nucl. Phys. B 323 (1989) 113 [INSPIRE].

[34] M. Ammon, A. Castro and N. Iqbal, Wilson Lines and Entanglement Entropy in Higher Spin Gravity, JHEP 10 (2013) 110 [arXiv:1306.4338] [INSPIRE].

[35] A. Campoleoni, T. Prochazka and J. Raeymaekers, A note on conical solutions in $3 D$ Vasiliev theory, JHEP 05 (2013) 052 [arXiv: 1303.0880] [INSPIRE].

[36] G. Felder, BRST Approach to Minimal Models, Nucl. Phys. B 317 (1989) 215 [Erratum ibid. B 324 (1989) 548] [INSPIRE].

[37] L.P. Kadanoff, Lattice Coulomb Gas Representations of Two-Dimensional Problems, J. Phys. A 11 (1978) 1399 [INSPIRE].

[38] B. Feigin and E. Frenkel, Quantization of the Drinfeld-Sokolov reduction, Phys. Lett. B 246 (1990) 75 [INSPIRE].

[39] L.F. Alday, D. Gaiotto and Y. Tachikawa, Liouville Correlation Functions from Four-dimensional Gauge Theories, Lett. Math. Phys. 91 (2010) 167 [arXiv:0906.3219] [INSPIRE].

[40] N. Wyllard, A(N-1) conformal Toda field theory correlation functions from conformal $N=2$ $\mathrm{SU}(N)$ quiver gauge theories, JHEP 11 (2009) 002 [arXiv:0907.2189] [INSPIRE].

[41] C. Córdova and D.L. Jafferis, Toda Theory From Six Dimensions, JHEP 12 (2017) 106 [arXiv: 1605.03997] [INSPIRE].

[42] R. Blumenhagen, D. Lüst and S. Theisen, Basic concepts of string theory, Springer-Verlag (2013). 